\title{
THE RIEMANN PROBLEM IN GAS DYNAMICS ${ }^{1}$
}

\author{
BY
}

\author{
RANDOLPH G. SMITH
}

\begin{abstract}
We consider the Riemann problem (R.P.) for the $3 \times 3$ system of gas dynamics equations in a single space variable. We assume that the specific internal energy $e=e(v, s) \quad(s=$ specific entropy, $v=$ specific volume) satisfies the usual hypotheses, $p_{v}<0, p_{v 0}>0, p_{s}>0\left(p=-e_{v}=\right.$ pressure); we also assume some reasonable hypotheses about the asymptotic behavior of $e$. We call functions $e$ satisfying these hypotheses energy functions.
\end{abstract}

THEOREM 1. For any initial data $\left(U_{l}, U_{r}\right)\left(U_{l}=\left(v_{l}, p_{l}, u_{l}\right), U_{r}=\left(v_{r}, p_{r}, u_{r}\right)\right.$, $u=$ flow velocity), the $R$. $P$. has a solution.

We introduce two conditions:

$$
\begin{array}{ll}
\frac{\partial}{\partial v} p(v, e)<\frac{p^{2}}{2 e} & (v, e>0), \\
\frac{\partial}{\partial v} e(v, p)>-\frac{p}{2} & (v, p>0) .
\end{array}
$$

THEOREM 2. (I) is necessary and sufficient for uniqueness of solutions of the $R$. P. Nonuniqueness persists under small perturbations of the initial data.

(I) is implied by the known condition

$$
\frac{\partial}{\partial v} e(v, p)>0 \quad(v, p>0)
$$

which holds for all usual gases. (I) implies (II). We construct energy functions $e$ that violate (II), that satisfy (II) but violate (I), and that satisfy (I) but violate (*).

In all solutions considered, the shocks satisfy the entropy condition and the Lax shock conditions.

1. Introduction. In this paper we investigate existence and uniqueness of solutions of the Riemann problem (R. P.), with arbitrary initial data, for the equations of compressible fluid flow in one space variable. The results depend on the energy function $e=e(\tau, S)(\tau=$ specific volume, $S=$ specific entropy). In addition to the assumptions normally made (see (1.2)-(1.7)), we always assume some physically reasonable limiting behaviors of the energy

Presented to the Society, November 3, 1975 under the title Solution of the Riemann problem in gas dynamics; received by the editors October 17, 1977.

AMS (MOS) subject classifications (1970). Primary 35L65, 76M05; Secondary 35L45, 35R05, 35Q99, 76J99, 76L05.

Key words and phrases. Conservation law, Riemann problem, gas dynamics, discontinuous data, compressible fluid flow, Hugoniot curve, shocks, rarefaction waves, contact discontinuity, ideal gases, Lax shock conditions, supersonic flow, equation of state, nonhomentropic gas flow.

${ }^{1}$ This paper comprises the results contained in a doctoral thesis submitted to the University of Michigan. 
and pressure functions near the boundaries of the domains on which they are defined (see (1.8)-(1.10)); ideal gases, for example, always satisfy these assumptions. We prove (\$3) that the R. P. always has a solution. However, solutions need not always be unique, even if they satisfy the entropy condition and the Lax shock conditions. Our analysis is based on the behavior of the Hugoniot curve $H$ (see (1.20)ff.) in the $(\tau, p)$-plane ( $p=$ pressure). It is sometimes assumed (see [1]) that $H$ is monotone in the sense that along $H$, one of the variables $\tau, p$ is a decreasing function of the other. This holds for polytropic gases, but need not hold for ideal gases. (See Ideal gases, §6.) It turns out that whether uniqueness fails, and if so how drastically, depends on how much $\boldsymbol{H}$ deviates from this monotonicity. We introduce three conditions on $e$ : WEAK, MEDIUM and STRONG (see Definition 1.4, Proposition 1.5), which we relate to the behavior of $H$ (see Lemmas 4.1, 4.2, 4.10, 4.11, 4.12 and Conventions 4.6, 4.7). WEAK and STRONG are already known ([2], [4]). The following implications hold:

$$
\text { polytropic gas } \Rightarrow \text { ideal gas } \Rightarrow \text { STRONG } \Rightarrow \text { MEDIUM } \Rightarrow \text { WEAK, }
$$

where all implications are proper. In $\S 4$ we prove that whenever MEDIUM holds, solutions of the R. P. are unique. However, whenever MEDIUM is violated, it is possible to construct initial data $\left(U_{l}, U_{r}\right)$ for which the R. P. has three distinct solutions; we call this particular situation type I nonuniqueness. We show that whenever WEAK fails, one cannot only obtain type I nonuniqueness, but also get nonuniqueness a different way, by a different construction; this is called type II nonuniqueness. The initial data that exhibit type II nonuniqueness also exhibit type I, and we then obtain five distinct solutions of the R. P. In $\$ 5$ we show that for both types of nonuniqueness the data can always be chosen so that the nonuniqueness is stable under perturbation of the initial data. In $\S 6$, we show by constructing various examples of energy functions, that all the possible situations abstractly considered in $\S \S 1-5$ do indeed occur, e.g., that the implications (*) are proper, and that it is possible to have five solutions of one R. P. For all solutions considered, the shocks always satisfy the entropy condition (see (1.18)) and the Lax shock conditions (see (1.19)).

The equations of compressible flow in one space variable (in Eulerian coordinates) are

$$
\begin{aligned}
\rho_{t}+(\rho u)_{x} & =0 \\
(\rho u)_{t}+\left(\rho u^{2}+p\right)_{x} & =0 \\
{\left[\rho\left(\frac{1}{2} u^{2}+e\right)\right]_{t}+\left[\rho u\left(\frac{1}{2} u^{2}+e\right)+u p\right]_{x} } & =0,
\end{aligned}
$$

where $t \geqslant 0$ is time, $x$ is position, $\rho$ is density, $p$ is pressure, $e$ is specific internal energy, and $u$ is flow velocity. We also let $S=$ specific entropy, 
$T=$ temperature, and $\tau=$ specific volume $\left(=\rho^{-1}\right)$. Here $e$ is assumed to be a $C^{3}$ function $e(\tau, S)$ of $\tau$ and $S, p=-e_{\tau}$, and $T=e_{S}$. The letter $g$ will always denote the function $-e_{\tau}$, i.e., $p=g(\tau, S)$. The function $e$ is assumed to satisfy the following

Standard assumptions.

$$
\begin{aligned}
e & >0, \\
p & >0 \quad(\text { i.e., } g>0), \\
T & >0 \quad\left(\text { i.e., } e_{S}>0\right), \\
g_{\tau} & <0, \\
g_{\tau \tau} & >0, \\
g_{S} & >0 .
\end{aligned}
$$

In addition, we shall always assume that $e$ satisfies the following

Limit assumptions.

$$
\begin{aligned}
& \lim _{S \rightarrow-\infty} g(\tau, S)=0, \quad \lim _{S \rightarrow+\infty} g(\tau, S)=+\infty, \\
& \lim _{S \rightarrow-\infty} e(\tau, S)=0, \quad \lim _{S \rightarrow+\infty} e(\tau, S)=+\infty, \\
& \lim _{\tau \rightarrow 0^{+}} e(\tau, p)=0 \text {. }
\end{aligned}
$$

Here the expression " $e(\tau, p)$ " requires definition. Suppose $\tau, p>0$. Then by (1.8), there is an $S$ such that $g(\tau, S)=p$, and by (1.7) $S$ is unique; we denote $S \equiv S(\tau, p)$. Then we define

$$
e(\tau, p) \equiv e(\tau, S(\tau, p))
$$

(whenever $p$ is understood to be a pressure rather than an entropy; cf. [1, $\S 64])$. We also define $g_{\tau}(\tau, p) \equiv g_{\tau}(\tau, S(\tau, p))$, etc.

REMARK. If we stipulate that $S>0$ and replace (1.8) and (1.9) by

$$
\lim _{S \rightarrow 0^{+}} g(\tau, S)=0, \quad \lim _{S \rightarrow+\infty} g(\tau, S)=+\infty
$$

and

$$
\lim _{S \rightarrow 0^{+}} e(\tau, S)=0, \quad \lim _{S \rightarrow+\infty} e(\tau, S)=+\infty,
$$

then it is easy to see that all results in this paper still hold, with the exception of that of $\S 6.3$; in this case an ideal gas (i.e., a function $e$ satisfying (1.2)-(1.7), $(1.8)^{\prime},(1.9)^{\prime},(1.10)$ and (6.75)) cannot exist.

Analogously, by (1.9) and (1.4) we can use $\rho, e$ (i.e., $\tau, e$ ) as independent variables so that $S$ and $p$ are functions of $(\rho, e)$. If we let $U=(\rho, u, e)$, then the equations (1.1) can be written as

$$
U_{t}+A(U) U_{x}=0
$$


for a $3 \times 3$ matrix $A(U)$ with eigenvalues $u-c, u$ and $u+c$, where $c$ (the sound speed) is defined by

$$
c^{2}=f_{\rho}
$$

where $f(\rho, S) \equiv g\left(\rho^{-1}, S\right)$.

The Riemann Problem (R. P.) (see [1], [4], [5]) for (1.1) or (1.12) is the initial-value problem for (1.1) with data

$$
U(x, 0)= \begin{cases}U_{l}, & \text { if } x<0 \\ U_{r}, & \text { if } x>0\end{cases}
$$

where $U_{l}=\left(\rho_{l}, u_{l}, e_{l}\right), U_{r}=\left(\rho_{r}, u_{r}, e_{r}\right)$ are arbitrary constant vectors.

By a solution of (1.1), (1.12) we mean a function $U(x / t)$ consisting of at most four constant states, $U_{l}, U_{1}, U_{3}, U_{r}$. Here $U_{l}$ (on the left) is connected to $U_{1}$ (on the right) by either a 1-shock or a 1-rarefaction wave, $U_{1}$ (left) is connected to $U_{3}$ (right) by a contact discontinuity, and $U_{3}$ (left) is connected to $U_{r}$ (right) by a 3-shock or a 3-rarefaction wave. (See [1] or [3] for the definition of these terms.)

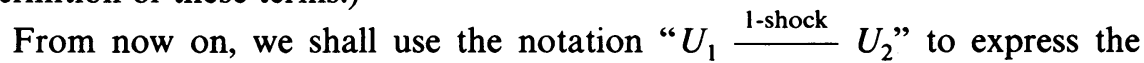
fact that $U_{1}$ (on the left) is, or can be, connected to $U_{2}$ (on the right) by a

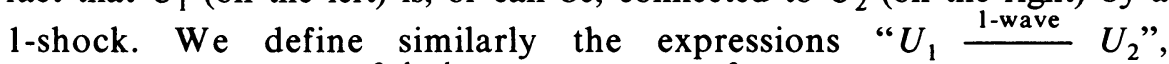
" $U_{1} \stackrel{\text { contact }}{ } U_{2}$ ", " $U_{1} \stackrel{\text { 3-shock }}{ } U_{2}$ " and " $U_{1} \stackrel{\text { 3-wave }}{ } U_{2}$ ", where "1-wave" (resp. "3-wave") is just an abbreviation for "1-rarefaction wave" (resp.

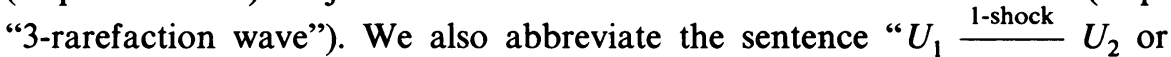
$U_{1} \stackrel{\text { l-wave }}{ } U_{2}$ " by " $U_{1} \frac{1}{2} U_{2}$ ", etc. Thus for a solution of the R. P. with initial data $\left(U_{l}, U_{r}\right)$ we must have $U_{1}$ and $U_{3}$ such that

$$
U_{l} \stackrel{1}{-} U_{1} \stackrel{\text { contact }}{=} U_{3} \stackrel{3}{-} U_{r} \text {. }
$$

Finally, since $(\rho, e)$ and $(\tau, p)$ determine each other, we can, and shall, describe states $U_{1}, U_{2}, \ldots$ by triples $\left(\tau_{1}, p_{1}, u_{1}\right),\left(\tau_{2}, p_{2}, u_{2}\right), \ldots$

By definition, $U_{1} \stackrel{\text { contact }}{\longrightarrow} U_{2}$ iff $u_{1}=u_{2}$ and $p_{1}=p_{2}$. For shocks, we have the following well-known result (see [1]):

Proposition 1.1. $U_{0}$ (left) can be connected to $U$ (right) by a shock with shock speed $s$ iff the following hold.

Jump conditions.

$$
\begin{aligned}
\rho v & =\rho_{0} v_{0} \equiv m \neq 0, \\
(\rho u) v+p & =\left(\rho_{0} u_{0}\right) v_{0}+p_{0}, \\
\rho\left(\frac{1}{2} u^{2}+e\right) v+p u & =\rho_{0}\left(\frac{1}{2} u_{0}^{2}+e_{0}\right) v_{0}+p_{0} u_{0},
\end{aligned}
$$

where $v \equiv u-s, v_{0} \equiv u_{0}-s$;

Entropy condition. 


$$
m S_{0} \leqslant m S .
$$

Moreover, the shock is a 1-shock (resp. 3-shock) iff $s<u, u_{0}$ (resp. $s>u, u_{0}$ ).

In this paper, shocks will also be required to satisfy the

Lax shock conditions.

$$
\begin{aligned}
& U_{0} \frac{\text { 1-shock }}{} U \Rightarrow v_{0}^{2}>c_{0}^{2} \text { and } v^{2}<c^{2} ; \\
& U \stackrel{\text { 3-shock }}{\stackrel{2}{U^{2}}} U_{0} \Rightarrow v_{0}^{2}>c_{0}^{2} \text { and } v^{2}<c^{2} .
\end{aligned}
$$

(See (1.13).)

The Hugoniot function with center $\left(\tau_{0}, p_{0}\right)$ is defined by

$$
H(\tau, p) \equiv e(\tau, p)-e\left(\tau_{0}, p_{0}\right)+\left(\tau-\tau_{0}\right) \frac{p+p_{0}}{2} \quad(\tau, p>0) .
$$

We define the Hugoniot curve $H$ with center $\left(\tau_{0}, p_{0}\right)$ by

$$
H \equiv\{(\tau, p) \mid H(\tau, p)=0\} \text {. }
$$

Let $U, U_{0}$ be constant states, and let $H$ have center $\left(\tau_{0}, p_{0}\right)$. For $a>0$, let $\mathcal{H}_{a}$ be the upper branch of the hyperbola, $\left(\tau_{0}-\tau\right)\left(p-p_{0}\right)=a$. Let $\mathcal{H}_{a}^{\prime}$ be the lower branch. (See Figure 1.) The importance of the Hugoniot curve lies in the following two facts.

Proposition 1.2.

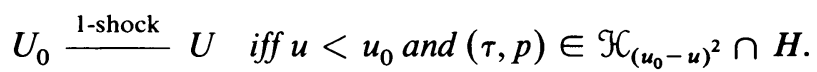

Proposition 1.3.

$$
U \stackrel{\text { 3-shock }}{ } U_{0} \quad \text { iff } u>u_{0} \text { and }(\tau, p) \in \mathcal{H}_{\left(u-u_{0}\right)^{2}} \cap H .
$$

These will be proved in $\$ 2$.

DEFINITION 1.4. $e=e(\tau, S)$ is an energy function iff it satisfies the Standard assumptions (1.2)-(1.7) and the Limit assumptions (1.8)-(1.10).

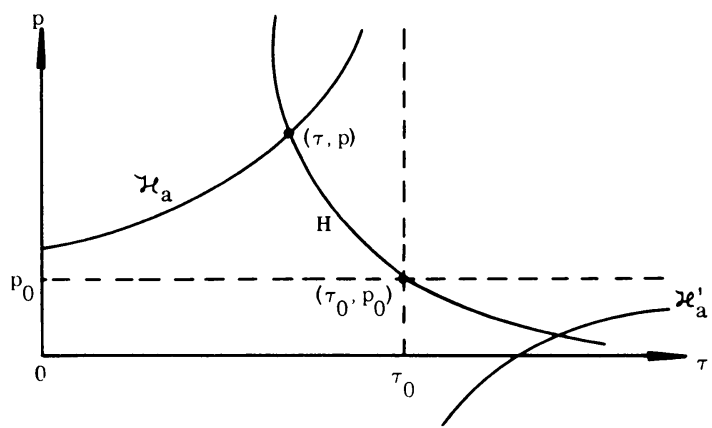

FIgURE 1. The hyperbola $H_{a} \cup H_{a}^{\prime}$ and a Hugoniot curve $H$. 
We introduce the following conditions on an energy function $e$ (see (1.11)):

$$
\begin{array}{lll}
\text { STRONG } & \frac{\partial}{\partial \tau} e(\tau, p) \geqslant 0 & (\tau, p>0), \\
\text { MEDIUM } & \left(\frac{\partial p}{\partial \tau}\right)_{e} \leqslant \frac{p^{2}}{2 e} & (\tau, e>0), \\
\text { WEAK } & \frac{\partial}{\partial \tau} e(\tau, p) \geqslant-\frac{p}{2} & (\tau, p>0) .
\end{array}
$$

Proposition 1.5. The conditions STRONG, MEDIUM and WEAK have the following equivalent formulations:

STRONG

$$
\frac{\partial}{\partial p} e(\tau, p) \geqslant \frac{p}{-g_{\tau}(\tau, p)} \quad(\tau, p>0)
$$

MEDIUM $\frac{\partial}{\partial p} e(\tau, p)>\frac{2 e(\tau, p)}{-g_{\tau}(\tau, p) \cdot(2 e(\tau, p) / p)+p} \quad(\tau, p>0)$,

$$
\text { WEAK } \quad \frac{\partial}{\partial p} e(\tau, p) \geqslant \frac{p}{-2 g_{\tau}(\tau, p)} \quad(\tau, p>0) \text {. }
$$

Proof. We have

$$
\frac{\partial}{\partial p} e(\tau, p)=\frac{\partial}{\partial p} e(\tau, S(\tau, p))=e_{S} \frac{\partial}{\partial p} S(\tau, p)=\frac{e_{S}}{g_{S}} .
$$

Here we have abbreviated $e_{S}(\tau, S(\tau, p))\left(=e_{S}(\tau, p)\right)$ by $e_{S}$; we shall do this often, e.g., $g_{\tau}=g_{\tau}(\tau, p)$. In particular

$$
\partial e(\tau, p) / \partial p>0 .
$$

Moreover

$$
\frac{\partial}{\partial \tau} e(\tau, p)=\frac{\partial}{\partial \tau} e(\tau, S(\tau, p))=e_{\tau}+e_{S} \frac{\partial}{\partial \tau} S(\tau, p)
$$

But $p=g(\tau, S(\tau, p))$, so taking $\partial / \partial \tau$ of both sides yields $0=g_{\tau}+g_{S} \partial S(\tau$, p) $/ \partial \tau$; hence

$$
\frac{\partial}{\partial \tau} e(\tau, p)=e_{\tau}+e_{S}\left(-\frac{g_{\tau}}{g_{S}}\right)
$$

so

$$
\frac{\partial}{\partial \tau} e(\tau, p)=-p+\left(-g_{\tau}\right) \frac{\partial}{\partial p} e(\tau, p)
$$

by (1.21). Solving (1.23) for $-g_{\tau}$, and substituting in the inequalities of Proposition 1.5, we obtain the result. 
polytropic

$\mathrm{H}$ is monotone

existence and uniqueness

MEDIUM or ideal

$\tau$ is a function of $p$ along $H$ $\forall a>0, \mathcal{K}_{a} \cap \mathrm{H}$ consists of one point

(H may not be monotone)

existence and uniqueness

WEAK and not-MEDIUM

$\tau$ is a function of $\mathrm{p}$ along $\mathrm{H}$

(*) $\exists \mathrm{a}>0 \ni \mathcal{H}_{\mathrm{a}} \cap \mathrm{H}$ contains more than one point

existence

(*) type I nonuniqueness

not-WEAK

(*) $\exists \mathrm{p}>0, \exists \tau_{1}, \tau_{2} \in\left(0, \tau_{0}\right) \ni$ $\left(\tau_{1}, p\right),\left(\tau_{2}, p\right) \in \mathrm{H}$

existence

(*) both type I and type II nonuniqueness
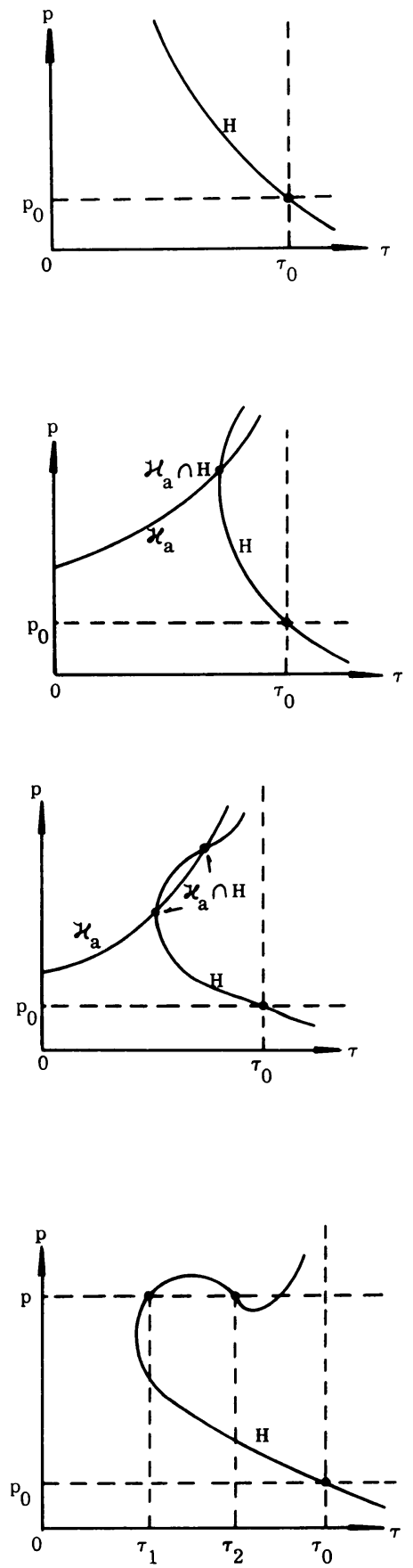

FIGURE 2. Hugoniot curves in different situations. 
Lemma 1.6. Let $\phi$ be defined on $(0,+\infty)$ with $\phi>0, \phi^{\prime}<0$ and $\phi^{\prime \prime}>0$. Then for every $\tau>0$,

$$
\phi(\tau)^{2}<-2 \phi^{\prime}(\tau) \int_{\tau}^{+\infty} \phi
$$

Proof. Straightforward.

Proposition 1.7. STRONG $\Rightarrow$ MEDIUM $\Rightarrow$ WEAK.

Proof. Apply Lemma 1.6 to the function $g(\cdot, S)$, note that

$$
\int_{\tau}^{+\infty} g(v, S) d v<e(\tau, S)
$$

and use Proposition 1.5.

We conclude with a rough summary, in table form, of what will be proved in the remainder of this paper regarding the connection between: (1), the conditions MEDIUM and WEAK; (2), the behavior of the Hugoniot curve $H$; and (3), existence and uniqueness of solutions of the R. P. The properties marked with a (*) are proved to hold for some choice of $\left(\tau_{0}, p_{0}\right)$ as center of $H$, or of $\left(U_{l}, U_{r}\right)$ as initial data; the others hold in general.

2. Properties of the Hugoniot curve. This section establishes some properties of $H$ that hold for all energy functions $e$, under no additional assumptions. We first summarize some known results due to Weyl; these are proved in [1, $\S 65]$.

Proposition 2.1 (WEYL). Let $H$ have center $\left(\tau_{0}, p_{0}\right)$ and let $\left(\tau_{1}, p_{1}\right) \in$ $H \backslash\left\{\left(\tau_{0}, p_{0}\right)\right\}$. Let $R$ be the line through $\left(\tau_{0}, p_{0}\right)$ and $\left(\tau_{1}, p_{1}\right)$, parametrized by

$$
p(s)=p_{0}+a s, \quad \tau(s)=\tau_{0}+b s,
$$

where $a=p_{1}-p_{0}, b=\tau_{1}-\tau_{0}$. Then

$$
\left.\frac{d}{d s} S(\tau(s), p(s))\right|_{s=0}>0 ;\left.\quad \frac{d}{d s} S(\tau(s), p(s))\right|_{s=1}<0 .
$$

Corollary 2.2. Let $H$ have center $\left(\tau_{0}, p_{0}\right)$ and let $\left(\tau_{1}, p_{1}\right) \in H \backslash\left\{\left(\tau_{0}, p_{0}\right)\right\}$. Let $L$ be the line through $\left(\tau_{0}, p_{0}\right)$ and $\left(\tau_{1}, p_{1}\right)$. Then $L$ cannot be tangent to $H$ at $\left(\tau_{1}, p_{1}\right)$; i.e., if the $C^{1}$ functions $\tau(t), p(t), t$ in a neighborhood of $t_{1}$, satisfy

$$
\left(\tau\left(t_{1}\right), p\left(t_{1}\right)\right)=\left(\tau_{1}, p_{1}\right), \quad(\tau(t), p(t)) \in H \quad \text { for all } t,
$$

then there cannot exist $\gamma \neq 0$ such that

$$
\left(\tau^{\prime}\left(t_{1}\right), p^{\prime}\left(t_{1}\right)\right)=\gamma\left(\tau_{1}-\tau_{0}, p_{1}-p_{0}\right) .
$$

Corollary 2.3. $d S \neq 0$ along $H$ except at $\left(\tau_{0}, p_{0}\right)$; i.e., if $\tau_{1}, p_{1}, t_{1}, \tau(t), p(t)$ are as in Corollary 2.2, and $\left(\tau^{\prime}\left(t_{1}\right), p^{\prime}\left(t_{1}\right)\right) \neq 0$, then

$$
\left.\frac{d}{d t} S(\tau(t), p(t))\right|_{t=t_{1}} \neq 0 \text {. }
$$


CoRollaRY 2.4. Whenever $\tau_{1}<\tau_{0}$ and $\left(\tau_{1}, p_{1}\right) \in H$,

$$
-g_{\tau}\left(\tau_{0}, S_{0}\right)<\frac{p_{1}-p_{0}}{\tau_{0}-\tau_{1}}<-g_{\tau}\left(\tau_{1}, S_{1}\right)
$$

thus if $U_{0} \stackrel{1 \text {-shock }}{ } U_{1}$ or $U_{1} \stackrel{\text { 3-shock }}{ } U_{0}$, then $v_{0}^{2}>c_{0}^{2}$ and $v_{1}^{2}<c_{1}^{2}$. (Cf. (1.19) and Propositions 1.1-1.3.)

The proofs that follow are long and tedious; hence we preface the formal proofs with an informal introduction. We define two subsets, $Q$ and $Q^{\prime}$, of the $(\tau, p)$-plane by

$$
\begin{aligned}
Q & =\left\{(\tau, p) \mid 0<\tau<\tau_{0} \text { and } p>p_{0}\right\}, \\
Q^{\prime} & =\left\{(\tau, p) \mid \tau>\tau_{0} \text { and } 0<p<p_{0}\right\},
\end{aligned}
$$

where $\left(\tau_{0}, p_{0}\right)$ is the center of the Hugoniot curve $H$. We prove that $\operatorname{grad} H \neq 0$ everywhere on $H$, and that $H \subset Q \cup Q^{\prime} \cup\left\{\left(\tau_{0}, p_{0}\right)\right\}$.

Definition 2.5. For all $\tau, p>0$, define

$$
\begin{gathered}
\theta=\theta(\tau, p)=\frac{p-p_{0}}{\tau_{0}-\tau} \quad \text { if } \tau \neq \tau_{0} ; \\
\theta_{0}=\theta\left(\tau_{0}, p_{0}\right)=-\left.\frac{d p}{d \tau}\right|_{\left(\tau_{0}, p_{0}\right)} \quad \text { along } H .
\end{gathered}
$$

Then we find that the function $\theta$ is $C^{1}$ on $H$; we plan to use $\theta$ as parameter along $H$. We shall be concerned only with the "compression branch" $H \cap$ $Q^{\text {cl }}$ of $H$. For all $\theta$, let $L_{\theta}$ be the line through $\left(\tau_{0}, p_{0}\right)$ with slope $-\theta$. We want to prove that along $H,(\tau, p)$ is a $C^{1}$ function of $\theta$; i.e., as $L_{\theta}$ sweeps through $Q$ in the clockwise direction, starting at $\theta_{0}$, there is always exactly one $(\tau(\theta)$, $p(\theta)) \in H \cap L_{\theta}$. (See Figure 3.) This is true locally by Corollary 2.2 and the fact that $\operatorname{grad} H \neq 0$ on $H$, since then $d \theta \neq 0$ along $H$. In particular, $H$ is represented by $(\tau(\theta), p(\theta))$ uniquely in a neighborhood of $\left(\tau_{0}, p_{0}\right)$. Here $(\tau(\theta)$, $p(\theta))$ is defined for $\theta$ in some neighborhood of $\theta_{0}$. We try to define $(\tau(\theta)$, $p(\theta)$ ) on the largest possible interval $\left[\theta_{0}, \theta_{1}\right)$. Any two such functions $\left(\tau_{1}(\theta)\right.$, $\left.p_{1}(\theta)\right),\left(\tau_{2}(\theta), p_{2}(\theta)\right)$ on $\left[\theta_{0}, \theta_{1}\right)$ must be the same, since otherwise there would be a first point $(\bar{\tau}, \vec{p})$ along $H$ at which the two curves begin to separate, which contradicts $\operatorname{grad} H \neq 0$ at $(\bar{\tau}, \bar{p})$. (See Figure 4.) Hence there is a maximal interval $\left[\theta_{0}, \theta_{\max }\right)$ on which such a function $(\tau(\theta), p(\theta))$ can be defined. Then we prove $\theta_{\max }=+\infty$ by showing that if not, then we can obtain an accumulation point in $Q$ of $(\tau(\theta), p(\theta))$ as $\theta \uparrow \theta_{\max }$, and using this, extend $(\tau(\theta), p(\theta))$ to some $\left[\theta_{0}, \theta_{1}\right), \theta_{1}>\theta_{\max }$. Thus we have a $C^{1}$ branch $H^{\prime} \subset H$ along which $(\tau, p)$ is a function of $\theta \in\left[\theta_{0},+\infty\right)$, and we also prove that along $H^{\prime}, p \rightarrow+\infty$ as $\theta \rightarrow+\infty$; moreover, for every $a>0, \mathcal{H}_{a} \cap H^{\prime}$ $\neq \varnothing$. (Cf. Figure 1.) Finally we show $H^{\prime}=H \cap Q^{\mathrm{cl}}$ as follows: we pick any $(\bar{\tau}, \bar{p}) \in H \cap Q$; we start with a representation $\left(\tau_{1}(\theta), p_{1}(\theta)\right)(\theta$ near $\bar{\theta} \equiv \theta(\bar{\tau}$, $\bar{p})$ ) of $H$ near $(\bar{\tau}, \bar{p})$; we extend $\left(\tau_{1}, p_{1}\right)$ to a maximal left interval of existence 
$\left(\theta_{\min }, \bar{\theta}\right]$; we then prove that this curve $J=\left\{\left(\tau_{1}(\theta), p_{1}(\theta)\right) \mid \theta \in\left(\theta_{\min }, \bar{\theta}\right]\right\}$ must intersect $H^{\prime}$, so $J \subset H^{\prime}$; hence $(\bar{\tau}, \bar{p}) \in H^{\prime}$.

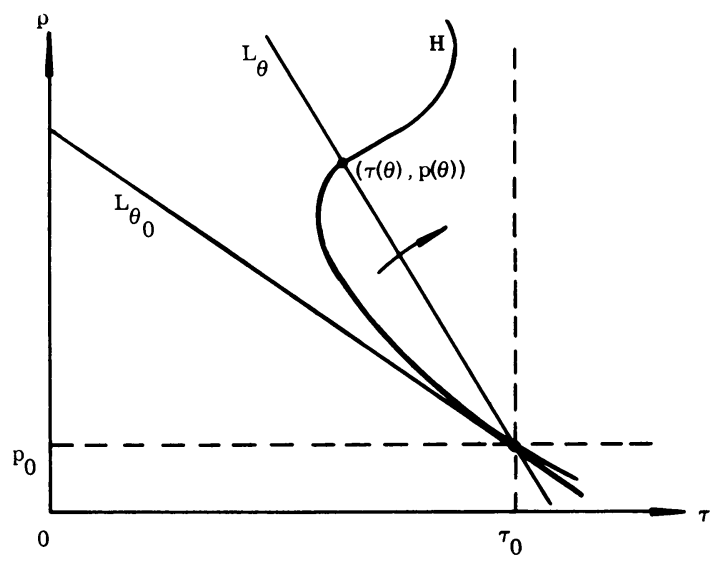

Figure 3. $(\tau, p)$ is a function of $\theta$ along $H$.

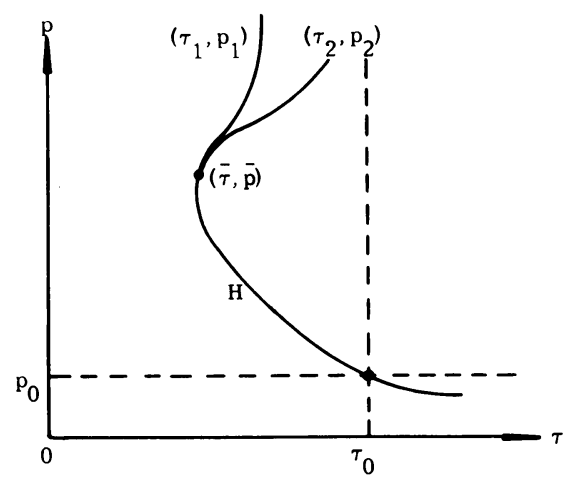

FIGURE 4. The images of $\left(\tau_{1}, p_{1}\right)$ and $\left(\tau_{2}, p_{2}\right)$ begin to separate at $(\bar{\tau}, \bar{p})$.

We hope the above will aid the reader in understanding and ignoring various tedious details of the proofs that follow.

Lemma 2.6. (a) $H_{\tau}(\tau, p)>0$ for $0<p \leqslant p_{0}$; (b) $H_{p}(\tau, p)>0$ for $\tau \geqslant \tau_{0}$; (c) $\operatorname{grad} H \neq 0$ everywhere on $H$.

Proof. Use (1.23) and Corollary 2.4.

Corollary 2.7. $H \subset Q \cup Q^{\prime} \cup\left\{\left(\tau_{0}, p_{0}\right)\right\}$.

Notation. For $x \in(-\infty,+\infty), \varepsilon>0$, let

$$
N_{\varepsilon}(x)=(x-\varepsilon, x+\varepsilon) \text {. }
$$


We know $[1, \S 65]$ that there exist $\delta, \sigma>0$ and a $C^{2}$ function $G$ on $N_{\delta}\left(\tau_{0}\right)$ such that

$$
G\left(\tau_{0}\right)=p_{0}, \quad G^{\prime}\left(\tau_{0}\right)=g_{\tau}\left(\tau_{0}, S_{0}\right), \quad G^{\prime \prime}\left(\tau_{0}\right)=g_{\tau \tau}\left(\tau_{0}, S_{0}\right),
$$

and for all $(\tau, p)$,

$$
(\tau, p) \in H \cap\left(N_{\delta}\left(\tau_{0}\right) \times N_{\sigma}\left(p_{0}\right)\right) \quad \text { iff } p=G(\tau) .
$$

Then Definition 2.5 implies

$$
\theta_{0} \equiv \theta\left(\tau_{0}, p_{0}\right)=-G^{\prime}\left(\tau_{0}\right)>0,
$$

by (2.3) and (1.5). Corollary 2.7 then yields

$$
\theta(\tau, p)>0 \text { for every }(\tau, p) \in H \text {. }
$$

Lemma 2.8. Define

$$
\theta(\tau)=\theta(\tau, G(\tau)) \quad \text { for } \tau \in N_{\delta}\left(\tau_{0}\right) .
$$

Then $\theta$ is $C^{1}$ and $\theta^{\prime}\left(\tau_{0}\right)<0$.

Proof. Straightforward.

LEMMA 2.9. $d \theta \neq 0$ along $H$.

Proof. $d \theta \neq 0$ at $\left(\tau_{0}, p_{0}\right)$ by Lemma 2.8 ; at $(\tau, p) \neq\left(\tau_{0}, p_{0}\right)$, use Corollary 2.2.

Definition 2.10. A $\theta$-parametrization $(\theta-p)$ on an interval $I$ is a $C^{1}$ vector function $\left(\tau^{0}, p^{0}\right)$ defined on $I$ such that

(a) $\left(\tau^{0}(\theta), p^{0}(\theta)\right) \in H(\theta \in I)$;

(b) $\theta\left(\tau^{0}(\theta), p^{0}(\theta)\right)=\theta(\theta \in I)$;

(c) if $\theta \in I$ and $\theta>\theta_{0}$, then $\left(\tau^{0}(\theta), p^{0}(\theta)\right) \in Q$.

(See Definition 2.5 for $\theta_{0}$.) A $\theta-p$ is centered $(c-\theta-p)$ if $\theta_{0} \in I$ implies $\left(\tau^{0}\left(\theta_{0}\right)\right.$, $\left.p^{0}\left(\theta_{0}\right)\right)=\left(\tau_{0}, p_{0}\right)$.

REMARK. The superscript " 0 " of ${ }^{\circ}\left(\tau^{0}, p^{0}\right)$ refers to the subscript in $\left(\tau_{0}, p_{0}\right)$, the center of $\boldsymbol{H}$. Later, this superscript will be varied to distinguish functions arising from Hugoniot curves with different centers.

LemMa 2.11. Let $(\bar{\tau}, \bar{p}) \in H \cap Q^{\mathrm{cl}}, \bar{\theta}=\theta(\bar{\tau}, \bar{p})$. Then there exist $\varepsilon_{1}, \varepsilon_{2}>0$, a $\theta-p\left(\tau^{*}, p^{*}\right)$ on $\left(\bar{\theta}-\varepsilon_{1}, \bar{\theta}+\varepsilon_{2}\right)$, and an open rectangle $R$ about $(\bar{\tau}, \bar{p})$, such that $\left(\tau^{*}(\bar{\theta}), p^{*}(\bar{\theta})\right)=(\bar{\tau}, \bar{p})$, and $\forall(\tau, p) \in H \cap R, \theta(\tau, p) \in\left(\bar{\theta}-\varepsilon_{1}, \bar{\theta}+\varepsilon_{2}\right)$ and $(\tau, p)=\left(\tau^{*}(\theta(\tau, p)), p^{*}(\theta(\tau, p))\right)$.

Proof. By Lemma 2.6(c), grad $H \neq 0$ at $(\bar{\tau}, \bar{p})$.

Case 1. $H_{p}(\bar{\tau}, \bar{p}) \neq 0$ or $(\bar{\tau}, \bar{p})=\left(\tau_{0}, p_{0}\right)$. Then $H_{p}(\bar{\tau}, \bar{p}) \neq 0$, so by the implicit function theorem, $\exists \delta, \sigma>0$ and a $C^{1}$ function $G$ on $N_{\delta}(\bar{\tau})$ such that for all $(\tau, p)$,

$$
(\tau, p) \in H \cap\left(N_{\delta}(\bar{\tau}) \times N_{\sigma}(\bar{p})\right) \text { iff } p=G(\tau) .
$$


Define

$$
\theta^{*}(\tau)=\theta(\tau, G(\tau)) \quad\left(\tau \in N_{\delta}(\bar{\tau})\right) .
$$

Then by Lemma 2.9 if $\bar{\tau}<\tau_{0}$, or by Lemma 2.8 if $\bar{\tau}=\tau_{0}, \theta^{*}$ is $C^{1}$ on a neighborhood of $\bar{\tau}$ and $\left(\theta^{*}\right)^{\prime}(\bar{\tau}) \neq 0$. Thus there exist $\delta_{1} \in(0, \delta]$ and $\varepsilon_{1}, \varepsilon_{2}>0$ such that

$$
\theta^{*} \text { is a } C^{1} \text { diffeomorphism of } N_{\delta_{1}}(\bar{\tau}) \text { onto }\left(\bar{\theta}-\varepsilon_{1}, \bar{\theta}+\varepsilon_{2}\right) .
$$

Let

$$
\tau^{*}=\left(\theta^{*}\right)^{-1}
$$

then $\tau^{*}$ is a $C^{1}$ diffeomorphism of $\left(\bar{\theta}-\varepsilon_{1}, \bar{\theta}+\varepsilon_{2}\right)$ onto $N_{\delta_{1}}(\bar{\tau})$. Let $p^{*}=$ $G \circ \tau^{*}$; then $p^{*}$ is $C^{1}$ on $\left(\bar{\theta}-\varepsilon_{1}, \bar{\theta}+\varepsilon_{2}\right)$. Since $\bar{p}=G(\bar{\tau}), \bar{\theta}=\theta^{*}(\bar{\tau})$, so $\left(\tau^{*}(\bar{\theta}), p^{*}(\bar{\theta})\right)=(\bar{\tau}, \bar{p})$. Clearly for $\theta \in\left(\bar{\theta}-\varepsilon_{1}, \bar{\theta}+\varepsilon_{2}\right)$ we have $\left(\tau^{*}(\theta)\right.$, $\left.p^{*}(\theta)\right)=\left(\tau^{*}(\theta), G\left(\tau^{*}(\theta)\right)\right) \in H$ and $\theta\left(\tau^{*}(\theta), p^{*}(\theta)\right)=\theta^{*}\left(\tau^{*}(\theta)\right)=\theta$. We claim that $\theta>\theta_{0}$ implies $\left(\tau^{*}(\theta), p^{*}(\theta)\right) \in Q$. If $(\bar{\tau}, \bar{p})=\left(\tau_{0}, p_{0}\right)$, then $\theta^{*}$ is decreasing on $N_{\delta_{1}}(\bar{\tau})$ by Lemma 2.8 and the fact that it is a diffeomorphism; hence $\tau^{*}$ is decreasing on $\left(\bar{\theta}-\varepsilon_{1}, \bar{\theta}+\varepsilon_{2}\right)$ and we are done by Lemma 2.7. If $(\bar{\tau}, \bar{p}) \neq\left(\tau_{0}, p_{0}\right)$, then $(\bar{\tau}, \bar{p}) \in Q$. Thus we can make the above $\delta$ and $\sigma$ (for (2.8)) sufficiently small that $N_{\delta}(\bar{\tau}) \times N_{\sigma}(\bar{p}) \subset Q$, and the claim is proved. Hence $\left(\tau^{*}, p^{*}\right)$ is a $\theta-p$ on $\left(\bar{\theta}-\varepsilon_{1}, \bar{\theta}+\varepsilon_{2}\right)$. Let $R=N_{\delta_{1}}(\bar{\tau}) \times N_{\sigma}(\bar{p})$. Let $(\tau$, $p) \in H \cap R$. Then $(\tau, p) \in N_{\delta}(\bar{\tau}) \times N_{\sigma}(\bar{p})$, so $p=G(\tau)$. Since $\tau \in N_{\delta_{1}}(\bar{\tau})$, we have

$$
\theta(\tau, p)=\theta^{*}(\tau) \in\left(\bar{\theta}-\varepsilon_{1}, \bar{\theta}+\varepsilon_{2}\right)
$$

and

$$
\begin{aligned}
& \tau^{*}(\theta(\tau, p))=\tau, \\
& p^{*}(\theta(\tau, p))=G\left(\tau^{*}(\theta(\tau, p))\right)=p .
\end{aligned}
$$

Case 2. $H_{\tau}(\bar{\tau}, \bar{p}) \neq 0$ and $(\bar{\tau}, \bar{p}) \neq\left(\tau_{0}, p_{0}\right)$. The proof is similar to Case 1 .

Corollary 2.12. Let $\left(\tau_{1}^{0}, p_{1}^{0}\right),\left(\tau_{2}^{0}, p_{2}^{0}\right)$ be $\theta-p$ 's on an interval $I$; let $\bar{\theta} \in I$ with $\left(\tau_{1}^{0}(\bar{\theta}), p_{1}^{0}(\bar{\theta})\right)=\left(\tau_{2}^{0}(\bar{\theta}), p_{2}^{0}(\bar{\theta})\right) \in Q^{\mathrm{cl}}$. Then $\left(\tau_{1}^{0}, p_{1}^{0}\right)=\left(\tau_{2}^{0}, p_{2}^{0}\right)$ on some neighborhood (in I) of $\bar{\theta}$.

An easy consequence of Corollary 2.12 is

Lemma 2.13. Let $\left(\tau_{1}^{0}, p_{1}^{0}\right),\left(\tau_{2}^{0}, p_{2}^{0}\right)$ be c- $\theta-p$ 's on $\left[\theta_{0}, \theta_{1}\right)$. Then $\left(\tau_{1}^{0}, p_{1}^{0}\right)=\left(\tau_{2}^{0}\right.$, $\left.p_{2}^{0}\right)$.

PROPOSITION 2.14. There exists a maximal right-open interval $\left[\theta_{0}, \theta_{\max }\right)$ on which a $c-\theta-p$ can be defined; i.e., there is a $c-\theta-p\left(\tau^{0}, p^{0}\right)$ on $\left[\theta_{0}, \theta_{\max }\right)$, and there cannot be ac- $\theta-p$ on any larger interval $\left[\theta_{0}, \theta_{1}\right), \theta_{1}>\theta_{\max }$. Here $\theta_{\max } \in\left(\theta_{0},+\infty\right]$. 
Proof. Let $\Theta=\left\{\theta_{1}>\theta_{0} \mid \exists a c-\theta-p\right.$ on $\left.\left[\theta_{0}, \theta_{1}\right)\right\}$. Let $\theta_{\max }=\sup \Theta$. With $\varepsilon_{2}$ as in Lemma 2.11 for $(\bar{\tau}, \bar{p})=\left(\tau_{0}, p_{0}\right), \theta_{0}+\varepsilon_{2} \in \Theta$; hence $\theta_{\max }>\theta_{0}$. We define $\left(\tau^{0}, p^{0}\right)$ on $\left[\theta_{0}, \theta_{\max }\right)$ as follows:

Let $\theta \in\left[\theta_{0}, \theta_{\max }\right)$. Choose some $\theta_{1} \in(\theta,+\infty) \cap \Theta$. $\exists a$ $c-\theta-p\left(\tau_{1}^{0}, p_{1}^{0}\right)$ on $\left[\theta_{0}, \theta_{1}\right)$. Let $\left(\tau^{0}(\theta), p^{0}(\theta)\right)=\left(\tau_{1}^{0}(\theta), p_{1}^{0}(\theta)\right)$.

By Lemma $2.13,\left(\tau^{0}, p^{0}\right)$ is well-defined by this process and is a $c-\theta-p$ on $\left[\theta_{0}\right.$, $\left.\theta_{\max }\right)$.

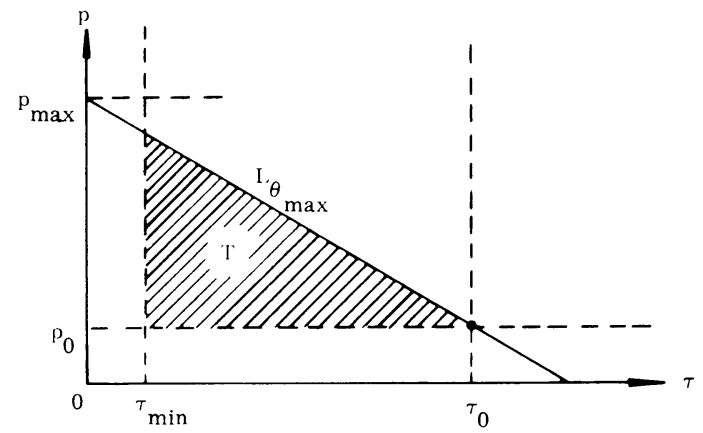

Figure 5. The region $T$.

Proposition 2.15. $\theta_{\max }=+\infty$.

PRoof. Suppose $\boldsymbol{\theta}_{\max }<+\infty$. Then $L_{\theta_{\max }}$ has $p$-intercept $p_{\max }=\tau_{0} \theta_{\max }+$ $p_{0}$. (See Figure 5.) Since $e\left(\tau_{0}, p_{0}\right)>0$, and $\lim _{\tau \rightarrow 0^{+}} e\left(\tau, p_{\max }\right)=0$ by (1.10), $\exists \tau_{\min }>0$ such that $\forall \tau>0, \tau \leqslant \tau_{\min }$ implies $e\left(\tau, p_{\max }\right)<e\left(\tau_{0}, p_{0}\right)$; we may assume $\tau_{\min } \leqslant \tau_{0}$. Now suppose $0<\tau<\tau_{\min }$ and $0<p<p_{\max }$. Then from (1.20) and (1.22), we get

$H(\tau, p) \leqslant e\left(\tau, p_{\max }\right)-e\left(\tau_{0}, p_{0}\right)+\left(\tau-\tau_{0}\right) \frac{p+p_{0}}{2}<\left(\tau-\tau_{0}\right) \frac{p+p_{0}}{2} \leqslant 0 ;$

thus

$$
\left[\tau \leqslant \tau_{\min } \text { and } p \leqslant p_{\max }\right] \Rightarrow(\tau, p) \notin H .
$$

Let $T$ be the open triangular region bounded by $L_{\theta_{\max }}$ and the lines $\left\{p=p_{0}\right\}$ and $\left\{\tau=\tau_{\min }\right\}$. (See Figure 5.) We assert that $\left(\tau^{\mathrm{max}}(\theta), p^{0}(\theta)\right) \in T$ $\forall \theta \in\left(\theta_{0}, \theta_{\text {max }}\right)$. For we know (Definition 2.10) that $\left(\tau^{0}(\theta), p^{0}(\theta)\right) \in H \cap Q$ and $\theta\left(\tau^{0}(\theta), p^{0}(\theta)\right)=\theta<\theta_{\max }$. Hence $\left(\tau^{0}(\theta), p^{0}(\theta)\right)$ lies below $L_{\theta_{\max }}$, which then implies $p^{0}(\theta) \leqslant p_{\max }$. From (2.10) we conclude $\tau^{0}(\theta)>\tau_{\min }$, and the assertion is proved.

Now choose a sequence $\left\{\theta_{n}\right\}$ from $\left(\theta_{0},+\infty\right)$ such that $\theta_{n} \uparrow \theta_{\max }$. Then $\left(\tau^{0}\left(\theta_{n}\right), p^{0}\left(\theta_{n}\right)\right) \in T^{\mathrm{cl}} \forall n$, so there exist a subsequence of $\left\{\boldsymbol{\theta}_{n}\right\}$, which we call $\left\{\theta_{n}\right\}$ again, and a point $(\bar{\tau}, \bar{p}) \in T^{\mathrm{cl}}$ such that

$$
\left(\tau^{0}\left(\theta_{n}\right), p^{0}\left(\theta_{n}\right)\right) \rightarrow(\bar{\tau}, \bar{p}) .
$$


Suppose $(\bar{\tau}, \bar{p})=\left(\tau_{0}, p_{0}\right)$; let $\varepsilon_{1}, \varepsilon_{2}, R$ be as in Lemma 2.11 . Let $N$ be so large that $\left(\tau^{0}\left(\theta_{n}\right), p^{0}\left(\theta_{n}\right)\right) \in R$ whenever $n \geqslant N$; then from Lemma 2.11 we conclude that $\forall n \geqslant N, \theta_{n}=\theta\left(\tau^{0}\left(\theta_{n}\right), p^{0}\left(\theta_{n}\right)\right) \in\left(\theta_{0}-\varepsilon_{1}, \theta_{0}+\varepsilon_{2}\right)$. But by Lemma 2.13 and (2.7)-(2.9) of the proof of Lemma 2.11, $\tau^{0}$ is a $C^{1}$ diffeomorphism on $\left(\theta_{0}-\varepsilon_{1}, \theta_{0}+\varepsilon_{2}\right)$, hence (strictly) monotone. Then since $\left\{\theta_{n}\right\}$ is increasing and $\left(\tau^{0}\left(\theta_{0}\right), p^{0}\left(\theta_{0}\right)\right)=\left(\tau_{0}, p_{0}\right)$, we cannot have $\tau^{0}\left(\theta_{n}\right) \rightarrow \tau_{0}$; this contradicts $(2.11)$. Therefore $(\bar{\tau}, \bar{p}) \neq\left(\tau_{0}, p_{0}\right)$. But since $H$ is closed, $(\bar{\tau}$, $\bar{p}) \in H$; then Corollary 2.7 gives $(\bar{\tau}, \bar{p}) \in Q$. Since $\theta$ is continuous on $Q$,

$$
\theta(\bar{\tau}, \bar{p})=\lim _{n \rightarrow \infty} \theta\left(\tau^{0}\left(\theta_{n}\right), p^{0}\left(\theta_{n}\right)\right)=\lim _{n \rightarrow \infty} \theta_{n}=\theta_{\max } ;
$$

hence Lemma 2.11 applies with $\bar{\theta}=\theta_{\max }$; let $\varepsilon_{1}, \varepsilon_{2}, R,\left(\tau^{*}, p^{*}\right)$ be as in Lemma 2.11 for $(\bar{\tau}, \bar{p}), \bar{\theta}$. Then $\left(\tau^{0}\left(\theta_{N}\right), p^{0}\left(\theta_{N}\right)\right) \in R \cap Q$ for some $N$, and we have

$$
\begin{gathered}
\theta_{N}=\theta\left(\tau^{0}\left(\theta_{N}\right), p^{0}\left(\theta_{N}\right)\right) \in\left(\theta_{\max }-\varepsilon_{1}, \theta_{\max }+\varepsilon_{2}\right), \\
\left(\tau^{0}\left(\theta_{N}\right), p^{0}\left(\theta_{N}\right)\right)=\left(\tau^{*}\left(\theta_{N}\right), p^{*}\left(\theta_{N}\right)\right) .
\end{gathered}
$$

Then $\left(\tau^{0}, p^{0}\right)$ and $\left(\tau^{*}, p^{*}\right)$ are $c-\theta-p$ 's on a neighborhood of $\theta_{N}$ that agree at $\theta_{N}$, so by Corollary $2.12,\left(\tau^{0}, p^{0}\right)=\left(\tau^{*}, p^{*}\right)$ on some neighborhood of $\theta_{N}$. Thus we may extend $\left(\tau^{0}, p^{0}\right)$ to $\left[\theta_{0}, \theta_{\max }+\varepsilon_{2}\right)$ by defining $\left(\tau^{0}, p^{0}\right)=\left(\tau^{*}, p^{*}\right)$ on $\left(\theta_{N}, \theta_{\max }+\varepsilon_{2}\right)$ and obtain a $C^{1}$ function, hence a $c-\theta-p$, on $\left[\theta_{0}, \theta_{\max }+\varepsilon_{2}\right)$, which contradicts the maximality of $\theta_{\max }$. Hence $\theta_{\max }=+\infty$.

Proposition 2.16. Let $(\bar{\tau}, \bar{p}) \in H \cap Q$. Then $(\bar{\tau}, \bar{p})=\left(\tau^{0}(\theta), p^{0}(\theta)\right)$ for some $\theta>\theta_{0}$.

Proof. Routine, using preceding results and methods of proof.

Thus, combining Propositions 2.14-2.16, we see that the entire curve $H \cap Q^{\mathrm{cl}}$ is given as a function of $\theta$ by the function $\left(\tau^{0}, p^{0}\right)$ on $\left[\theta_{0},+\infty\right)$.

Lemma 2.17. Along $H \cap Q^{\mathrm{cl}}, S$ increases with increasing $\theta$.

Proof. We know [1, §65] that near $\left(\tau_{0}, p_{0}\right), S$ increases along $H$ with decreasing $\tau$. Then by Lemma 2.8, $S$ increases along $H \cap Q^{\mathrm{cl}}$ with increasing $\theta$ for $\theta$ near $\theta_{0}$. Lemma 2.11 and its proof imply $\left(\left(\tau^{0}\right)^{\prime}(\theta),\left(p^{0}\right)^{\prime}(\theta)\right) \neq 0$ for $\theta>\theta_{0}$; hence

$$
\frac{d}{d \theta} S\left(\tau^{0}(\theta), p^{0}(\theta)\right) \neq 0 \text { for } \theta>\theta_{0},
$$

by Corollary 2.3. Then we must have

$$
\frac{d}{d \theta} S\left(\tau^{0}(\theta), p^{0}(\theta)\right)>0 \text { for } \theta>\theta_{0}
$$

hence $\theta \rightarrow S\left(\tau^{0}(\theta), p^{0}(\theta)\right)$ is increasing on $\left[\theta_{0},+\infty\right)$.

Let us remark that our assumptions (1.8) and (1.9) imply

$$
\lim _{p \rightarrow 0^{+}} e(\tau, p)=0 \quad \forall \tau>0 .
$$


Using this, Lemma 2.6 and Corollary 2.7 , it is easy to prove

LEMMA 2.18. $H \cap\left(Q^{\prime}\right)^{\mathrm{cl}}$ is given by a decreasing $C^{1}$ function $\tau=V(p)$ from $\left(0, p_{0}\right]$ onto $\left[\tau_{0}, \tau_{\max }\right)$, where

$$
\tau_{\max }=\tau_{0}+2 e\left(\tau_{0}, p_{0}\right) / p_{0} .
$$

CoROllaRY 2.19. Along $H \cap\left(Q^{\prime}\right)^{\mathrm{cl}}, S$ increases with increasing $p$.

Proof. Similar to that of Lemma 2.17, using Corollary 2.3, the function $G$ of Lemma 2.8, and Lemma 2.18.

We can now give the

Proof of Propositions 1.2 and 1.3. We prove Proposition 1.2; Proposition 1.3 is similar.

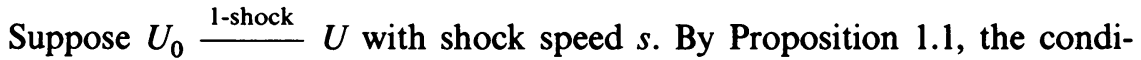
tions (1.15)-(1.18) hold. It is known [1, §§54, 55, 59, 64] that (1.15)-(1.17) imply

$$
(\tau, p) \in\left(\mathcal{H}_{\left(u_{0}-u\right)^{2}} \cup \mathcal{K}_{\left(u_{0}-u\right)^{2}}^{\prime}\right) \cap H,
$$

where $H, \mathcal{H}, \mathcal{H}^{\prime}$ have center $\left(\tau_{0}, p_{0}\right)$. Proposition 1.1 also gives $v, v_{0}>0$. Then (1.18) yields $S>S_{0}$ (where $S_{0}=S\left(\tau_{0}, p_{0}\right), S=S(\tau, p)$ ). From Corollary 2.19 we then conclude $(\tau, p) \notin\left(Q^{\prime}\right)^{\mathrm{cl}}$; hence $(\tau, p) \in \mathcal{F}_{\left(u_{0}-u\right)^{2}} \cap H$. In particular $(\tau, p) \in Q$, so $\tau<\tau_{0}$; then (1.15) yields $v<v_{0}$, so $u<u_{0}$.

Now suppose $u<u_{0}$ and $(\tau, p) \in \mathcal{K}_{\left(u_{0}-u\right)^{2}} \cap H$. If $s$ is to be the shock speed, then (1.15) is satisfied iff

$$
s=\left(\rho u-\rho_{0} u_{0}\right) /\left(\rho-\rho_{0}\right) .
$$

Using this value of $s$, one shows easily, by reversing the arguments of [1] cited above, that $U_{0}, U$ and $s$ also satisfy (1.16) and (1.17). Since $(\tau, p) \in \mathcal{H}_{\left(u_{0}-u\right)^{2}}$, $\rho>\rho_{0}$ and $p>p_{0}$. Then

$$
s=\frac{\rho u-\rho_{0} u_{0}}{\rho-\rho_{0}}<\frac{\rho u-\rho_{0} u}{\rho-\rho_{0}}=u .
$$

So $s<u, u_{0}$, and $m>0$. But $S>S_{0}$ by Lemma 2.17 (and Propositions 2.14-2.16, etc.), since ( $\tau, p) \in H \cap Q$; thus (1.18) holds, and $U_{0} \stackrel{\text { 1-shock }}{ } U$.

Now recall that in this paper all shocks are required to satisfy the Lax shock conditions (1.19). Thus for the purposes of this paper, the proof is not complete until (1.19) is proved. But (1.19) is a simple consequence of Corollary 2.4 and the fact that $\rho>\rho_{0}$ and $(\tau, p) \in H$.

Observe that the above proof also establishes

Proposition 2.20. Shocks always satisfy (1.19).

LEMMA 2.21. $\lim _{\theta \rightarrow+\infty} p^{0}(\theta)=+\infty$. 
Proof. Suppose not. Then we can find $M>0$ and a sequence $\left\{\theta_{n}\right\}$ from $\left(\theta_{0},+\infty\right)$ such that $\theta_{n} \rightarrow+\infty$ and $p^{0}\left(\theta_{n}\right) \leqslant M \forall n$. We have, for each $n$,

$$
\theta_{n}=\theta\left(\tau^{0}\left(\theta_{n}\right), p^{0}\left(\theta_{n}\right)\right)=\frac{p^{0}\left(\theta_{n}\right)-p_{0}}{\tau_{0}-\tau^{0}\left(\theta_{n}\right)} \leqslant \frac{M-p_{0}}{\tau_{0}-\tau^{0}\left(\theta_{n}\right)},
$$

since $\left(\tau^{0}\left(\theta_{n}\right), p^{0}\left(\theta_{n}\right)\right) \in Q$. (See Definition 2.10(c).) Since $\theta_{n} \rightarrow+\infty$, we must have $\tau^{0}\left(\theta_{n}\right) \rightarrow \tau_{0}$. There exist a subsequence of $\left\{\theta_{n}\right\}$, which we call $\left\{\theta_{n}\right\}$ again, and a number $\bar{p} \in\left[p_{0}, M\right]$ such that $p^{0}\left(\theta_{n}\right) \rightarrow \bar{p}$. Suppose $\bar{p}=p_{0}$. Let $R$ be as in Lemma 2.11 for $\left(\tau_{0}, p_{0}\right), \theta_{0}$. Then since $\left(\tau^{0}\left(\theta_{n}\right), p^{0}\left(\theta_{n}\right)\right) \rightarrow\left(\tau_{0}, \bar{p}\right)=\left(\tau_{0}, p_{0}\right)$, $\left(\tau^{0}\left(\theta_{n}\right), p^{0}\left(\theta_{n}\right)\right) \in R$ for arbitrarily large $\theta_{n}$, a contradiction. Hence $\bar{p}>p_{0}$. But since $H$ is closed, it follows that $\left(\tau_{0}, \vec{p}\right) \in H$, which contradicts Corollary 2.7.

LeMma 2.22. For every $a>0, \mathcal{H}_{a} \cap H \neq \varnothing$. In other words, the function $a^{0}(\theta), \theta \in\left[\theta_{0},+\infty\right)$ defined by

$$
a^{0}(\theta)=\sqrt{\left(\tau_{0}-\tau^{0}(\theta)\right)\left(p^{0}(\theta)-p_{0}\right)}
$$

assumes all nonnegative values.

Proof. Suppose $\exists a>0$ such that $\forall \theta \geqslant \theta_{0},\left(\tau^{0}(\theta), p^{0}(\theta)\right)$ lies to the right of $\mathcal{H}_{a}$, i.e.,

$$
\tau^{0}(\theta) \geqslant \tau_{0}-a /\left(p^{0}(\theta)-p_{0}\right) \quad\left(\theta \geqslant \theta_{0}\right) .
$$

Then from (1.20) and (2.13),

$$
\begin{aligned}
e\left(\tau^{0}(\theta), p^{0}(\theta)\right)-e\left(\tau_{0}, p_{0}\right) & =\left(\tau_{0}-\tau^{0}(\theta)\right) \frac{p^{0}(\theta)+p_{0}}{2} \\
& \leqslant \frac{a}{2} \frac{p^{0}(\theta)+p_{0}}{p^{0}(\theta)-p_{0}} \rightarrow \frac{a}{2} \quad \text { as } \theta \rightarrow+\infty,
\end{aligned}
$$

by Lemma 2.21. Hence $e\left(\tau^{0}(\theta), p^{0}(\theta)\right)$ is bounded above for $\theta \geqslant \theta_{0}$.

Choose $\theta_{1} \geqslant \theta_{0}$ so large that $L_{\theta_{1}} \cap \mathcal{H}_{a}$ is a point $\left(\tau_{1}, p_{1}\right) \in Q$, i.e., $\tau_{1}>0$. Then $\forall \theta \geqslant \theta_{1},\left(\tau^{0}(\theta), p^{0}(\theta)\right)$ lies above $L_{\theta_{1}}$ and to the right of $\mathcal{H}_{a}$; thus

$$
\tau^{0}(\theta) \geqslant \tau_{1} \quad\left(\theta \geqslant \theta_{1}\right) .
$$

Let $S^{0}(\theta)=S\left(\tau^{0}(\theta), p^{0}(\theta)\right)\left(\theta \geqslant \theta_{0}\right)$ and suppose $\exists M>0$ such that $\forall \theta>\theta_{0}, S^{0}(\theta) \leqslant M$. By Lemma 2.17, $S^{0}(\theta)>S\left(\tau_{0}, p_{0}\right) \forall \theta>\theta_{0}$. Thus $\forall \theta>\theta_{1},\left(\tau^{0}(\theta), S^{0}(\theta)\right) \in\left[\tau_{1}, \tau_{0}\right] \times\left[S\left(\tau_{0}, p_{0}\right), M\right]$. But $g$ is bounded above on $\left[\tau_{1}, \tau_{0}\right] \times\left[S\left(\tau_{0}, p_{0}\right), M\right]$, so $p^{0}(\theta)=g\left(\tau^{0}(\theta), S^{0}(\theta)\right)$ is bounded above for $\theta \geqslant \theta_{1}$, which contradicts Lemma 2.21. Therefore $S^{0}(\theta)$ assumes arbitrarily large values for $\theta \geqslant \theta_{0}$.

Finally, observe that $\forall \theta \geqslant \theta_{0}$,

$$
e\left(\tau^{0}(\theta), p^{0}(\theta)\right)=e\left(\tau^{0}(\theta), S^{0}(\theta)\right) \geqslant e\left(\tau_{0}, S^{0}(\theta)\right),
$$


by (1.3). But the left member of (2.21) is bounded above, whereas by (1.9) the right member is not, yielding a contradiction.

3. Existence of solutions. In this section we prove that for any energy function $e$ (see Definition 1.4), the R. P. with arbitrary initial data $\left(U_{l}, U_{r}\right)$ always has a solution. In order to do this, it is sometimes necessary, as usual, to allow a "void" constant state $(\rho, e, p=0)$, corresponding to a region in which there is no gas. (See [5], and Theorem 3.5, below.)

We first use the results of $\$ 2$ to investigate shocks. Throughout this section we assume that we are given arbitrary fixed initial data $\left(U_{l}, U_{r}\right)$. We define

$$
S_{1}=\left\{U \mid U_{l} \frac{1 \text {-shock }}{U}\right\}, \quad S_{3}=\left\{U \mid U \stackrel{\text { 3-shock }}{ } U_{r}\right\} .
$$

Recall $U_{l}=\left(\tau_{l}, p_{l}, u_{l}\right), U_{r}=\left(\tau_{r}, p_{r}, u_{r}\right)$. Let $H^{l}, Q^{l}, \theta^{l}, \theta_{l}, L_{\theta}^{l},\left(\tau^{l}, p^{l}\right), \mathcal{H}_{a}^{l}$ and $a^{l}$ be the $H, Q, \theta, \theta_{0}, L_{\theta},\left(\tau^{0}, p^{0}\right), \mathcal{F}_{a}$ and $a^{0}$ of $\S 2$ referred to the center $\left(\tau_{l}, p_{l}\right)$ instead of $\left(\tau_{0}, p_{0}\right)$; thus, for example, $H^{l}$ is the Hugoniot curve with center $\left(\tau_{l}\right.$, $\left.p_{l}\right), \theta^{\prime}(\tau, p)=\left(p-p_{l}\right) /\left(\tau_{l}-\tau\right)$ for $\tau \neq \tau_{l}$, and

$$
a^{l}(\theta)=\sqrt{\left(\tau_{l}-\tau^{l}(\theta)\right)\left(p^{l}(\theta)-p_{l}\right)} \quad\left(\theta \in\left[\theta_{l},+\infty\right)\right) .
$$

Define $H^{r}, Q^{r}, \theta^{r}, \theta_{r}, L_{\theta}^{r},\left(\tau^{r}, p^{r}\right), \mathcal{H}_{a}^{r}$ and $a^{r}$ similarly relative to $\left(\tau_{r}, p_{r}\right)$. Then we have

Proposition 3.1. $S_{1}=\left\{U_{1}(\theta) \mid \theta>\theta_{l}\right\}$ where $U_{1}(\theta)=\left(\tau^{l}(\theta), p^{l}(\theta), u^{l}(\theta)\right)$ $\left(\theta \geqslant \theta_{l}\right)$, and $u^{l}(\theta)=u_{l}-a^{l}(\theta)\left(\theta \geqslant \theta_{l}\right)$. Thus $\left(\tau^{l}, p^{l}\right)$ is $C^{1}$ on $\left[\theta_{l},+\infty\right)$ with $\left(\tau^{l}\left(\theta_{l}\right), p^{l}\left(\theta_{l}\right)\right)=\left(\tau_{l}, p_{l}\right)$, and

$$
\left(\tau^{l}(\theta), p^{l}(\theta)\right) \in H^{l} \cap Q^{l} \cap L_{\theta}^{l} \text { for } \theta>\theta_{l} .
$$

Moreover, $\left(p^{l}, u^{l}\right)$ is continuous and injective on $\left[\theta_{l},+\infty\right),\left(p^{l}\left(\theta_{l}\right), u^{l}\left(\theta_{l}\right)\right)=$ $\left(p_{l}, u_{l}\right), \lim _{\theta \rightarrow+\infty} p^{l}(\theta)=+\infty$, and $u^{l}(\theta)$ is not bounded below as $\theta \rightarrow+\infty$.

Proof. Propositions 2.14 and 2.15 imply the existence of a $c-\theta-p\left(\tau^{l}, p^{l}\right)$ on $\left[\theta_{l},+\infty\right)$. Thus by Definition 2.10, $\left(\tau^{l}, p^{l}\right)$ is $C^{l},\left(\tau^{l}\left(\theta_{l}\right), p^{l}\left(\theta_{l}\right)\right)=\left(\tau_{l}, p_{l}\right)$, and (3.1) holds. By Lemma $2.21, \lim _{\theta \rightarrow+\infty} p^{l}(\theta)=+\infty$; by Lemma $2.22, u^{l}(\theta)$ is not bounded below as $\theta \rightarrow+\infty$. Also $u^{l}$ is clearly continuous; hence so is $\left(p^{l}, u^{l}\right)$. It is easily verified that $S_{1}=\left\{U_{1}(\theta) \mid \theta>\theta_{l}\right\}$, and that $\left(p^{l}, u^{l}\right)$ is injective.

Similarly we have

Proposition 3.2. $S_{3}=\left\{U_{3}(\theta) \mid \theta>\theta_{r}\right\}$ where $U_{3}(\theta)=\left(\tau^{r}(\theta), p^{r}(\theta), u^{r}(\theta)\right)$, $u^{r}(\theta)=u_{r}+a^{r}(\theta)\left(\theta \geqslant \theta_{r}\right)$. Thus $\left(\tau^{r}, p^{r}\right)$ is $C^{1}$ on $\left[\theta_{r},+\infty\right)$ with $\left(\tau^{r}\left(\theta_{r}\right)\right.$, $\left.p^{r}\left(\theta_{r}\right)\right)=\left(\tau_{r}, p_{r}\right)$, and

$$
\left(\tau^{r}(\theta), p^{r}(\theta)\right) \in H^{r} \cap Q^{r} \cap L_{\theta}^{r} \quad \text { for } \theta>\theta_{r} .
$$

Moreover, $\left(p^{r}, u^{r}\right)$ is continuous and injective on $\left[\theta_{r},+\infty\right),\left(p^{r}\left(\theta_{r}\right), u^{r}\left(\theta_{r}\right)\right)=$ $\left(p_{r}, u_{r}\right), \lim _{\theta \rightarrow+\infty} p^{r}(\theta)=+\infty$, and $u^{r}(\theta)$ is not bounded above as $\theta \rightarrow+\infty$. 
We now study the rarefaction wave curves. Let

$$
W_{1}=\left\{U \mid U_{l} \stackrel{\text { 1-wave }}{ } U\right\}, \quad W_{3}=\left\{U \mid U \stackrel{\text { 3-wave }}{ } U_{r}\right\} \text {. }
$$

Proposition 3.3. Along $W_{1}, U$ is a continuous function $U_{1}(p)=\left(\tau_{1}(p), p\right.$, $\left.u_{l}(p)\right)$ of $p \in\left(0, p_{l}\right] ; u_{1}$ is continuous and decreasing and maps $\left(0, p_{l}\right]$ onto $\left[u_{l}\right.$, $\left.u_{\max }\right)$ for some $u_{\max } \in\left(u_{l},+\infty\right]$.

Proof. Suppose $v_{1}$ and $v_{2}$ are independent 1-Riemann invariants. Then by [3], $U \in W_{1}$ iff

$$
v_{1}(U)=v_{1}\left(U_{l}\right), \quad v_{2}(U)=v_{2}\left(U_{l}\right), \quad u-c \geqslant u_{l}-c_{l} .
$$

Moreover, using equations equivalent to (1.1) with unknowns $\rho, u, S$, we see [3] that we may take

$$
v_{1}(U)=S, \quad v_{2}(U)=u+h(\rho, S),
$$

where

$$
h(\rho, S)=\int_{\rho_{l}}^{\rho} \bar{\rho}^{-1} \sqrt{f_{\rho}(\bar{\rho}, S)} d \bar{\rho},
$$

so that

$$
\partial h(\rho, S) / \partial \rho=c / \rho .
$$

(Here $f(\rho, S) \equiv g\left(\rho^{-1}, S\right)$; see (1.13).) Then by (3.3) and (3.4), $U \in W_{1}$ iff

$$
\begin{aligned}
S & =S_{l}, \\
u+h\left(\rho, S_{l}\right) & =u_{l}+h\left(\rho_{l}, S_{l}\right), \\
u-c & \geqslant u_{l}-c_{l} .
\end{aligned}
$$

By (3.7) and (3.5), $u$ is a decreasing function of $\rho$ along $W_{1}$. Its domain is restricted by (3.8) as follows. Since $g_{\tau \tau}(\tau, S)=\rho^{3}\left[\rho f_{\rho \rho}(\rho, S)+2 f_{\rho}(\rho, S)\right]$, (1.7) implies

$$
\rho f_{\rho \rho}+2 f_{\rho}>0 \text {. }
$$

Then along $W_{1}$ we have

$$
\frac{d}{d \rho}(u-c)=-\frac{\partial}{\partial \rho} h\left(\rho, S_{l}\right)-\frac{\partial}{\partial \rho} \sqrt{f_{\rho}\left(\rho, S_{l}\right)}=-\frac{1}{2 \rho c}\left(2 f_{\rho}+\rho f_{\rho \rho}\right)<0 .
$$

So assuming (3.6) and (3.7), (3.8) is equivalent to $\rho \in\left(0, \rho_{l}\right]$; thus along $W_{1}, u$ is a continuous, decreasing function of $\rho$, defined for $\rho \in\left(0, \rho_{l}\right]$. Since $p=g\left(\rho^{-1}, S_{l}\right)$ along $W_{1},(1.2),(1.3)$ and (1.5) imply that along $W_{1}, p$ is a continuous, increasing function of $\rho$ mapping $\left(0, \rho_{l}\right]$ onto $\left(0, \rho_{l}\right]$. It follows that along $W_{1}, \tau$ is a continuous, decreasing function $\tau_{1}(p)$ of $p \in\left(0, p_{l}\right]$, and $u$ is a continuous, decreasing function $u_{l}(p)$ of $p \in\left(0, p_{l}\right]$, with range $\left[u_{l}, u_{\max }\right)$ for some $u_{\max } \in\left(u_{l},+\infty\right]$. Thus along $W_{1}, U$ is a continuous function $U_{1}(p)=\left(\tau_{1}(p), p, u_{1}(p)\right)$ of $p \in\left(0, p_{l}\right]$ as desired. 
Similarly we have

Proposition 3.4. Along $W_{3}, U$ is a continuous function $U_{3}(p)=\left(\tau_{3}(p), p\right.$, $\left.u_{3}(p)\right)$ of $p \in\left(0, p_{r}\right] ; u_{3}$ is continuous and increasing and maps $\left(0, p_{r}\right]$ onto $\left(u_{\min }\right.$, $\left.u_{r}\right]$ for some $u_{\min } \in\left[-\infty, u_{r}\right)$.

THEOREM 3.5. If $u_{\min }<u_{\max }$, then the $R$. $P$. (with initial data $\left(U_{l}, U_{r}\right)$ ) has a solution. If $u_{\min } \geqslant u_{\max }$, then the $R$. P. has no solution; however, if we allow $\rho$, $p, e=0$, then the R.P. has a solution.

Proof. Let $C_{1}=S_{1} \cup W_{1}, C_{3}=S_{3} \cup W_{3}$. Thus

$$
C_{1}=\left\{U \mid U_{l} \stackrel{1}{-} U\right\}, \quad C_{3}=\left\{U \mid U \stackrel{3}{-} U_{r}\right\} .
$$

Case 1. $u_{\max }>u_{\min }$. Let $\bar{C}_{1}$ (resp. $\bar{C}_{3}$ ) be the projection of $C_{1}$ (resp. $C_{3}$ ) onto the $(p, u)$-plane. Then from Propositions 3.1-3.4 it is easy to see that $\bar{C}_{1}$ and $\bar{C}_{3}$ must intersect, i.e., there is a $(\bar{p}, \bar{u}) \in \bar{C}_{1} \cap \bar{C}_{3}$. Hence $\exists \tau_{1}, \tau_{3}>$ $0 \ni\left(\tau_{1}, \bar{p}, \vec{u}\right) \in C_{1}$ and $\left(\tau_{3}, \bar{p}, \vec{u}\right) \in C_{3}$. Then the R. P. has the solution

$$
U_{l} \stackrel{1}{-}\left(\tau_{1}, \bar{p}, \bar{u}\right) \stackrel{\text { contact }}{ }\left(\tau_{3}, \bar{p}, \bar{u}\right) \stackrel{3}{-} U_{r} .
$$

Case 2. $u_{\max } \leqslant u_{\min }$. If the problem had a solution, then there would exist states $U_{1}$ and $U_{3}$ such that

$$
U_{l} \stackrel{1}{-} U_{1} \stackrel{\text { contact }}{=} U_{3} \stackrel{3}{-} U_{r} .
$$

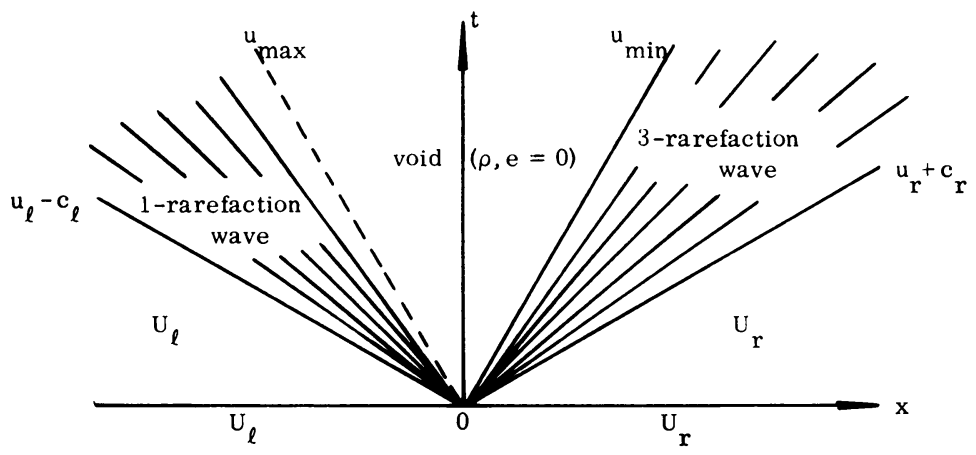

Figure 6. The case of the vacuum.

These relations imply $U_{1} \in C_{1}, u_{1}=u_{3}$ and $U_{3} \in C_{3}$; then, using Propositions 3.1-3.4, also $u_{1}<u_{\max }$ and $u_{3}>u_{\min }$, a contradiction. Hence there is no solution. But if we allow $\rho, p, e=0$ (corresponding to a vacuum), then we can connect $U_{l}$ and $U_{r}$ each by a (complete) rarefaction wave to a constant "void" state ( $\rho, e=0, u$ undefined). (See [5], and Figure 6.) To see that these two 
waves do not overlap, we note that $u-c<u_{\max }$ on $W_{1}$, and $u+c>u_{\min }$ on $W_{3}$. Thus our "solution" is

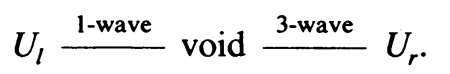

4. Uniqueness and nonuniqueness of solutions. In this section we show that the condition MEDIUM is necessary and sufficient for uniqueness of solutions of the R. P. (See Theorem 4.5 and Theorem 4.13.) We first prove the sufficiency. To obtain uniqueness, we replace Propositions 3.1 and 3.2 by Propositions 4.3 and 4.4, which assert that when MEDIUM holds, the curves $S_{1}$ and $S_{3}$ have a simpler form: along $\overline{S_{1}}$ (the projection of $S_{1}$ onto the ( $p$, $u$ )-plane), $u$ is a decreasing function of $p$; along $\overline{S_{3}}, u$ is an increasing function of $p$. Then the curves $\overline{C_{1}}$ and $\overline{C_{3}}$ can intersect in at most one point. (See Figure 8.)

LEMma 4.1. Suppose e satisfies WEAK. Then along $H, \tau=V(p)$, where $V$ is a $C^{1}$ function defined on $(0,+\infty)$.

Proof. Straightforward.

When WEAK holds, we define

$$
a_{0}(p)=\sqrt{\left(\tau_{0}-V(p)\right)\left(p-p_{0}\right)} \quad(p>0)
$$

LEMMA 4.2. Suppose e satisfies MEDIUM. Then

$$
\frac{d}{d p} a_{0}(p)>0 \quad\left(p>p_{0}\right)
$$

Proof. Let $H^{0}$ denote the Hugoniot curve with center $\left(\tau_{0}, p_{0}\right)$. Since WEAK holds, we have $\tau=V^{0}(p)$ along $H^{0}$ by Lemma 4.1; also,

$$
H_{\tau}(\tau, p)>0 \text {. }
$$

As in (4.1), we define $a_{0}$ by

$$
a_{0}(p)=\sqrt{\left(\tau_{0}-V^{0}(p)\right)\left(p-p_{0}\right)} \quad(p>0) .
$$

Then

$$
\frac{d}{d p} V^{0}(p)=-\frac{H_{p}\left(V^{0}(p), p\right)}{H_{\tau}\left(V^{0}(p), p\right)} .
$$

We write $\tau=V^{0}(p)$; then, using (1.23), we obtain $\frac{d}{d p} V^{0}(p)$

$$
=\frac{1}{2 a_{0}(p)}\left[\left(\tau_{0}-\tau\right)+\left(p-p_{0}\right) \frac{(\partial / \partial p) e(\tau, p)-\left(\tau_{0}-\tau\right) / 2}{\left(-g_{\tau}\right)(\partial / \partial p) e(\tau, p)-\left(p-p_{0}\right) / 2}\right]
$$


But

$$
\left(-g_{\tau}\right) \frac{\partial}{\partial p} e(\tau, p)-\frac{p-p_{0}}{2}>0
$$

by (4.2) and (1.23), so

$$
\frac{d}{d p} a_{0}(p)>0 \Leftrightarrow \frac{\partial}{\partial p} e(\tau, p)>\frac{\left(p-p_{0}\right)\left(\tau_{0}-\tau\right)}{\left(-g_{\tau}\right)\left(\tau_{0}-\tau\right)+\left(p-p_{0}\right)} .
$$

We also have

which we shall use later.

$$
\frac{d}{d p} a_{0}(p)<0 \Leftrightarrow \frac{\partial}{\partial p} e(\tau, p)<\frac{\left(p-p_{0}\right)\left(\tau_{0}-\tau\right)}{\left(-g_{\tau}\right)\left(\tau_{0}-\tau\right)+\left(p-p_{0}\right)},
$$

Let $\left(\tau_{1}, p_{1}\right) \in H^{0} \cap Q$ (see (2.1)); then $\tau_{1}=V^{0}\left(p_{1}\right)$. We must prove

By (4.3), it suffices to prove

$$
\left.\frac{d}{d p} a_{0}(p)\right|_{p=p_{1}}>0 \text {. }
$$

$$
\frac{\partial}{\partial p} e\left(\tau_{1}, p_{1}\right)>\frac{\left(p_{1}-p_{0}\right)\left(\tau_{0}-\tau_{1}\right)}{\left(-g_{\tau}\left(\tau_{1}, p_{1}\right)\right)\left(\tau_{0}-\tau_{1}\right)+\left(p_{1}-p_{0}\right)} .
$$

Let $H^{1}$ be the Hugoniot curve with center $\left(\tau_{1}, p_{1}\right)$, and let $\tau=V^{1}(p)$ along $H^{1}$ as in Lemma 4.1. Then $\left(\tau_{0}, p_{0}\right) \in H^{1}$, so $\tau_{0}=V^{1}\left(p_{0}\right)$. Since $\tau_{0}>\tau_{1}$ and $p_{0}<p_{1}$, Lemma 2.18 gives $\tau_{0}=V^{1}\left(p_{0}\right) \in\left[\tau_{1}, \tau_{\max }^{1}\right)$, where $\tau_{\max }^{1}=\tau_{1}+$ $\left[2 e\left(\tau_{1}, p_{1}\right) / p_{1}\right]$. Hence

$$
0<\tau_{0}-\tau_{1}<2 e\left(\tau_{1}, p_{1}\right) / p_{1} .
$$

Also,

$$
0<p_{1}-p_{0}<p_{1}
$$

Now observe that

$$
\frac{\left(p_{1}-p_{0}\right)\left(\tau_{0}-\tau_{1}\right)}{\left(-g_{\tau}\left(\tau_{1}, p_{1}\right)\right)\left(\tau_{0}-\tau_{1}\right)+\left(p_{1}-p_{0}\right)}=f\left(\tau_{0}-\tau_{1}, p_{1}-p_{0}\right),
$$

where $f$ is the function

$$
f(x, y)=x y /(a x+b y) \quad(x, y>0),
$$

where $a=-g_{\tau}\left(\tau_{1}, p_{1}\right)>0, b=1>0$. But $f$ satisfies $\partial f / \partial x>0, \partial f / \partial y>0$; hence from (4.6) and (4.7) we get

$$
\begin{aligned}
f\left(\tau_{0}-\tau_{1}, p_{1}-p_{0}\right) & <f\left(\frac{2 e\left(\tau_{1}, p_{1}\right)}{p_{1}}, p_{1}\right) \\
& =\frac{2 e\left(\tau_{1}, p_{1}\right)}{\left(-g_{\tau}\left(\tau_{1}, p_{1}\right)\right) 2 e\left(\tau_{1}, p_{1}\right) / p_{1}+p_{1}} \leqslant \frac{\partial}{\partial p} e\left(\tau_{1}, p_{1}\right),
\end{aligned}
$$

by MEDIUM and Proposition 1.5. This, together with (4.8), proves (4.5). 
Proposition 4.3. Suppose e satisfies MEDIUM. Then along $S_{1}, U$ is a continuous function $U_{1}(p)=\left(\tau_{1}(p), p, u_{1}(p)\right)$ of $p>p_{l}$, and $u_{1}$ is a continuous, decreasing function mapping $\left(p_{l},+\infty\right)$ onto $\left(-\infty, u_{l}\right)$.

Proof. Let $H^{l}$ be the Hugoniot curve with center $\left(\tau_{l}, p_{l}\right)$; let $\tau=V^{l}(p)$ along $H^{l}$ as in Lemma 4.1, and define

$$
a_{l}(p)=\sqrt{\left(\tau_{l}-V^{l}(p)\right)\left(p-p_{l}\right)} \quad(p>0) .
$$

Define $\tau_{1}=V^{l}$ and $u_{1}(p)=u_{l}-a_{l}(p)\left(p>p_{l}\right)$.

Similarly we have

Proposition 4.4. Suppose e satisfies MEDIUM. Then along $S_{3}, U$ is a continuous function $U_{3}(p)=\left(\tau_{3}(p), p, u_{3}(p)\right)$ of $p>p_{r}$, and $u_{3}$ is a continuous, increasing function mapping $\left(p_{r},+\infty\right)$ onto $\left(u_{r},+\infty\right)$.

THEOREM 4.5. Suppose e satisfies MEDIUM. Then the R. P. always has a unique solution (in the sense of Theorem 3.5).

Proof. We are given arbitrary initial data $\left(U_{l}, U_{r}\right)$. As in Theorem 3.5, let $C_{1}=S_{1} \cup W_{1}, C_{3}=S_{3} \cup W_{3}$. Combining Propositions 3.3 and 4.3, we have

Along $C_{1}, U$ is a continuous function $U_{1}(p)=\left(\tau_{1}(p), p\right.$, $\left.u_{1}(p)\right)$ of $p>0 ; u_{1}$ is a continuous, decreasing function mapping $(0,+\infty)$ onto $\left(-\infty, u_{\max }\right)$, for some $u_{\max } \in\left(u_{l}\right.$, $+\infty]$.

(See Figure 7.) Similarly, Propositions 3.4 and 4.4 give

Along $C_{3}, U$ is a continuous function $U_{3}(p)=\left(\tau_{3}(p), p\right.$, $\left.u_{3}(p)\right)$ of $p>0 ; u_{3}$ is a continuous, increasing function mapping $(0,+\infty)$ onto $\left(u_{\min },+\infty\right)$, for some $u_{\min } \in[-\infty$, $\left.u_{r}\right)$.

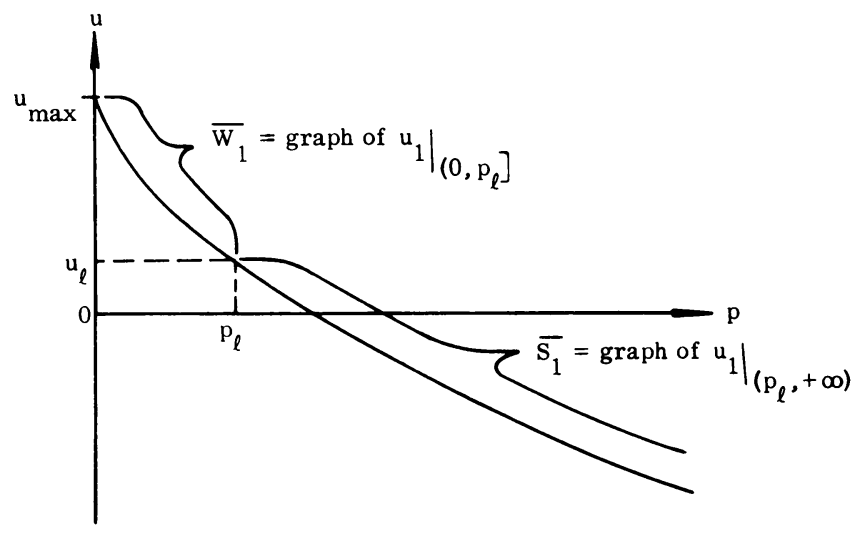

FIGURE 7. $u$ is a decreasing function of $p$ along $\bar{C}_{1}$. 
Case 1. $u_{\max }>u_{\min }$. Let $\overline{C_{1}}$ (resp. $\overline{C_{3}}$ ) be the projection of $C_{1}$ (resp. $C_{3}$ ) onto the $(p, u)$-plane. Then $\overline{C_{1}}$ is the graph of $u_{1}$, and $\overline{C_{3}}$ is the graph of $u_{3}$. A solution of R.P. is uniquely determined by states $\overline{U_{1}}, \overline{U_{3}}$ such that

$$
U_{l} \stackrel{1}{-} \overline{U_{1}} \stackrel{\text { contact }}{=} \overline{U_{3}} \stackrel{3}{-} U_{r} \text {. }
$$

For such states $\overline{U_{1}}, \overline{U_{3}}$ we must have $\overline{U_{1}} \in C_{1}, \overline{U_{3}} \in C_{3}$ and $\left(\overline{p_{1}}, \overline{u_{1}}\right)=\left(\overline{p_{3}}, \overline{u_{3}}\right)$. In this case, denote $\overline{p_{1}}$ and $\overline{p_{3}}$ by $\bar{p}$; then by (4.9) and (4.10), $\bar{p}$ uniquely determines $\overline{U_{1}}$ and $\overline{U_{3}}$, and $u_{1}(\bar{p})=u_{3}(\bar{p})$. But $u_{1}$ is decreasing and $u_{3}$ is increasing, so $u_{1}-u_{3}$ is decreasing; hence $u_{1}(\bar{p})=u_{3}(\bar{p})$ can hold for at most one $\bar{p}$. (See Figure 8 .) Thus there can be at most one solution of the R. P.

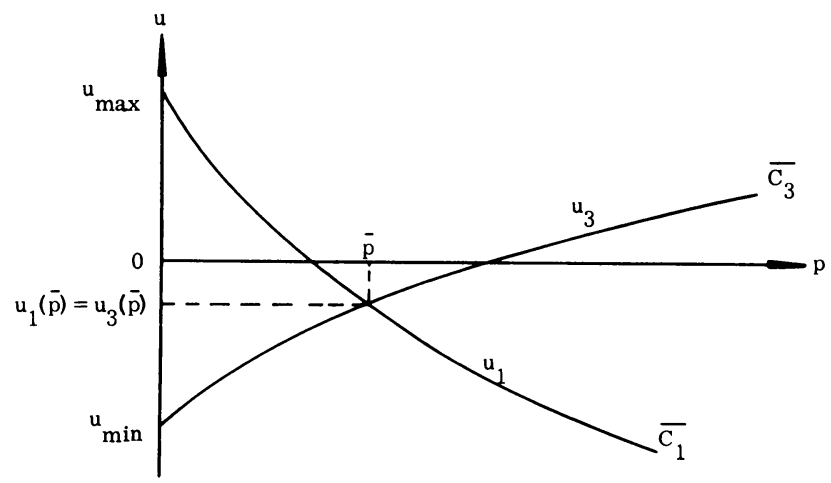

FIGURE 8. The strict monotonicity of $\bar{C}_{1}$ and $\bar{C}_{3}$ assures uniqueness.

Here it is necessary to stipulate that in the case $u_{\max }>u_{\min }$, we do not admit the void state $(\rho, e=0, u$ undefined). (See Theorem 3.5, Case 2.)

Case 2. $u_{\max } \leqslant u_{\min }$. Clearly there is only one way to connect $U_{l}$ (left) to the void state on the right by a complete 1-rarefaction wave; similarly for

$$
\text { void } \stackrel{\text { 3-wave }}{\longrightarrow} U_{r} \text {. }
$$

We turn now to nonuniqueness. We begin by showing two different ways in which a property of the Hugoniot curve allows one to construct initial data $\left(U_{l}, U_{r}\right)$ for which the R. P. has more than one solution.

Convention 4.6. $H$ will always denote the Hugoniot curve with center $\left(\tau_{0}\right.$, $\left.p_{0}\right)$. Similarly, we shall always denote

$Q=\left(0, \tau_{0}\right) \times\left(p_{0},+\infty\right)$,

$\theta=\left(p-p_{0}\right) /\left(\tau_{0}-\tau\right)\left(\tau \neq \tau_{0}\right)$

$\mathcal{H}_{a}=$ upper branch of hyperbola $\left(\tau_{0}-\tau\right)\left(p-p_{0}\right)=a$,

$L_{\bar{\theta}}=$ line through $\left(\tau_{0}, p_{0}\right)$ with slope $-\bar{\theta}(\bar{\theta}>0)$.

(See, for example, Lemma 4.10, below.)

First, suppose we have two distinct points $\left(\tau_{1}, p_{1}\right),\left(\tau_{2}, p_{2}\right)$ and $a>0$ such 
that

$$
\left(\tau_{i}, p_{i}\right) \in \mathcal{H}_{a^{2}} \cap H
$$

for $i=1$, 2. (See Figure 9.) Let $u_{l}$ be arbitrary and let $U_{l}=\left(\tau_{0}, p_{0}, u_{l}\right)$, $U_{r}=\left(\tau_{0}, p_{0}, u_{l}-2 a\right)$. Then (4.11) and Propositions 1.2 and 1.3 yield the following two solutions of the R. P. with data $\left(U_{l}, U_{r}\right)$ :

$$
\begin{aligned}
& U_{l} \frac{1 \text {-shock }}{}\left(\tau_{1}, p_{1}, u_{l}-a\right) \stackrel{\text { 3-shock }}{U_{r},} \\
& U_{l} \stackrel{\text { 1-shock }}{ }\left(\tau_{2}, p_{2}, u_{l}-a\right) \stackrel{\text { 3-shock }}{=} U_{r} .
\end{aligned}
$$

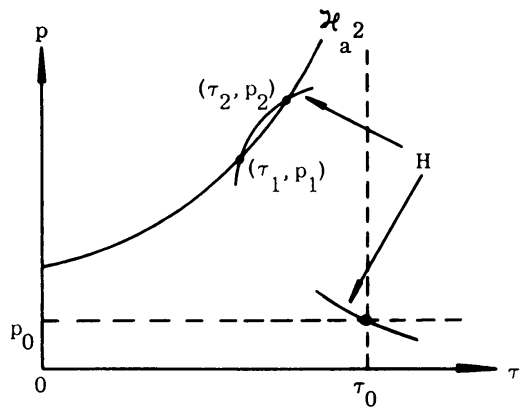

Figure 9. The behavior of $H$ that gives rise to type I nonuniqueness.

Clearly, if we have $n$ distinct points $\left(\tau_{1}, p_{1}\right), \ldots,\left(\tau_{n}, p_{n}\right)$ that satisfy (4.11) for $i=1, \ldots, n$, then we obtain $n$ distinct solutions of the R. P. with data $\left(U_{l}\right.$, $\left.U_{r}\right)$. Motivated by the above, we make

DEFINITION 4.7. We say that type I nonuniqueness holds for $\left(U_{l}, U_{r}\right)$ if $U_{l}$ and $U_{r}$ have the same thermodynamic variables $\left(\tau_{0}, p_{0}\right)$ and there exist distinct $\left(\tau_{1}\right.$, $\left.p_{1}\right)$ and $\left(\tau_{2}, p_{2}\right)$ that satisfy (4.11), where $2 a=u_{l}-u_{r}$; then the R. P. with data $\left(U_{l}, U_{r}\right)$ has the two distinct solutions (4.12) and (4.13), which we call type I solutions. The (type I) nonuniqueness is $n$-fold if there exist $n$ (type I) solutions.

Now suppose we have $\bar{p}>p_{0}$ and $0<\tau_{1}<\tau_{2}<\tau_{0}$ such that

$$
\left(\tau_{1}, \bar{p}\right),\left(\tau_{2}, \bar{p}\right) \in H \text {. }
$$

(See Figure 10.) Let $a$ and $b$ satisfy

$$
\left(\tau_{1}, \bar{p}\right) \in \mathcal{H}_{b^{2}}, \quad\left(\tau_{2}, \bar{p}\right) \in \mathcal{H}_{a^{2}}
$$

(e.g., $\left.a=\sqrt{\left(\tau_{0}-\tau_{2}\right)\left(\bar{p}-p_{0}\right)}\right)$; then $b>a>0$. Let $u_{l}$ be arbitrary and define $U_{l}=\left(\tau_{0}, p_{0}, u_{l}\right), \quad U_{r}=\left(\tau_{0}, p_{0}, u_{l}-(a+b)\right)$. Then by (4.14), (4.15), Propositions 1.2 and 1.3 , and the meaning of contact discontinuity, the R. P. with data $\left(U_{l}, U_{r}\right)$ has the following two solutions:

$$
\begin{aligned}
& U_{l} \stackrel{\text { 1-shock }}{\longrightarrow}\left(\tau_{1}, \bar{p}, u_{l}-b\right) \stackrel{\text { contact }}{ }\left(\tau_{2}, \bar{p}, u_{l}-b\right) \stackrel{\text { 3-shock }}{U_{r}} U_{\text {, }} \\
& U_{l} \stackrel{\text { 1-shock }}{ }\left(\tau_{2}, \bar{p}, u_{l}-a\right) \stackrel{\text { contact }}{\longrightarrow}\left(\tau_{1}, \bar{p}, u_{l}-a\right) \stackrel{\text { 3-shock }}{=} U_{r} \text {. }
\end{aligned}
$$




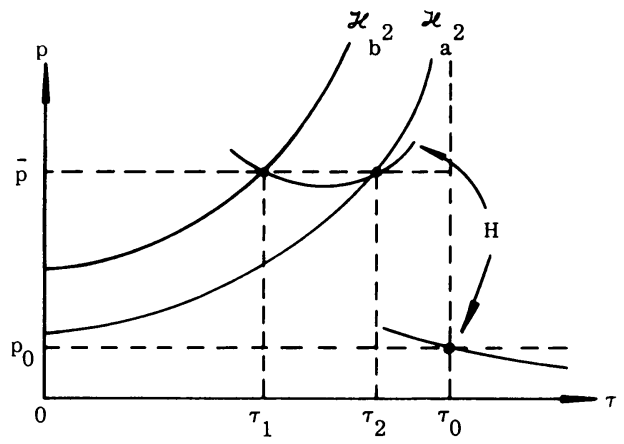

FIgURE 10. The behavior of $H$ that gives rise to type II nonuniqueness.

Definition 4.8. We say that type II nonuniqueness holds for $\left(U_{l}, U_{r}\right)$ if $U_{l}$ and $U_{r}$ have the same thermodynamic variables $\left(\tau_{0}, p_{0}\right)$ and there exist $\bar{p}>p_{0}, \tau_{1}, \tau_{2} \in\left(0, \tau_{0}\right)$ with $\tau_{1}<\tau_{2}, a>0$ and $b>0$ such that $u_{l}-u_{r}=a+$ $b$ and (4.14) and (4.15) hold; then the R. P. with data $\left(U_{l}, U_{r}\right)$ has the two solutions (4.16) and (4.17), which we call type II solutions.

We now prove that MEDIUM is necessary for uniqueness. Some of the lemmas below are stronger than necessary; these strengthened statements will be used in $\$ 5$ to obtain stability.

Convention 4.9. We shall henceforth of ten use a more informal notation, in which all derivatives are assumed to be taken along $H$. Here are two examples:

$$
\left.\frac{d \tau}{d \theta}\right|_{\left(\tau_{1}, \bar{p}\right)}=\left.\frac{d}{d \theta} \tau^{0}(\theta)\right|_{\theta=\theta_{1}},
$$

where $\tau_{1}, \bar{p}$ are as above and $\theta_{1}=\theta\left(\tau_{1}, \bar{p}\right) ; d \tau / d p=V^{\prime}(p)$ when WEAK holds and $V$ is as in Lemma 4.1.

LEMMA 4.10. Suppose e satisfies WEAK, but violates MEDIUM. Then there exist $\left(\tau_{0}, p_{0}\right)$, and $\left(\tau_{1}, p_{1}\right) \in H \cap Q$, such that

$$
\left.\frac{d a}{d p}\right|_{\left(\tau_{1}, p_{1}\right)}<0,\left.\quad \frac{d p}{d \theta}\right|_{\left(\tau_{1}, p_{1}\right)}>0 .
$$

(See Conventions 4.6 and 4.9; here, and often in what follows, we also denote $\left.a=\sqrt{\left(\tau_{0}-\tau\right)\left(p-p_{0}\right)}\right)$

Proof. Since $e$ violates MEDIUM, then by Proposition 1.5 there exists $\left(\tau_{1}\right.$, $\left.p_{1}\right)$ such that

$$
\frac{\partial}{\partial p} e\left(\tau_{1}, p_{1}\right)<\frac{2 e\left(\tau_{1}, p_{1}\right)}{-g_{\tau}\left(\tau_{1}, p_{1}\right) \cdot\left(2 e\left(\tau_{1}, p_{1}\right) / p_{1}\right)+p_{1}} .
$$


Let $H^{1}$ be the Hugoniot curve with center $\left(\tau_{1}, p_{1}\right)$. We obtain the first inequality by choosing $\left(\tau_{0}, p_{0}\right) \in H^{1}$ with $p_{0}>0$ sufficiently small, using Lemma 2.18 and (4.4). The second inequality is clear.

Now suppose $(\tau, p)$ satisfies $H(\tau, p)=0$ and $H_{\tau}(\tau, p) \neq 0$. Then $\tau$ is a function of $p$ along $H$, near $(\tau, p)$; hence $d \tau / d p$ exists and

$$
\frac{d \theta}{d p}=\frac{\left(\tau_{0}-\tau\right)+\left(p-p_{0}\right) d \tau / d p}{\left(\tau_{0}-\tau\right)^{2}}
$$

if $\tau \neq \tau_{0}$; by Lemma 2.9, $d \theta / d p \neq 0$; thus $d p / d \theta$ exists and is nonzero. In particular, if $(\tau, p) \in H, p>p_{0}$ and $H_{\tau}(\tau, p) \neq 0$, then by (4.19) the following hold:

$$
\begin{aligned}
& \frac{d p}{d \theta} \neq 0, \\
& \frac{d p}{d \theta}>0 \Leftrightarrow \frac{d \tau}{d p}>\frac{\tau-\tau_{0}}{p-p_{0}}, \\
& \frac{d p}{d \theta}<0 \Leftrightarrow \frac{d \tau}{d p}<\frac{\tau-\tau_{0}}{p-p_{0}} \Rightarrow \frac{d \tau}{d p}<0 .
\end{aligned}
$$

LEMMA 4.11. Suppose e violates WEAK. Then there exist $\left(\tau_{0}, p_{0}\right)$, and $\left(\tau_{2}\right.$, p) $\in H \cap Q$, such that

$$
\left.\frac{d \tau}{d p}\right|_{\left(\tau_{2}, \bar{p}\right)}<0,\left.\quad \frac{d p}{d \theta}\right|_{\left(\tau_{2}, \bar{p}\right)}<0
$$

Proof. Since WEAK fails, $\exists\left(\tau_{2}, \bar{p}\right)$ such that

$$
\frac{\partial}{\partial p} e\left(\tau_{2}, \bar{p}\right)<\frac{\bar{p}}{-2 g_{\tau}\left(\tau_{2}, \bar{p}\right)} .
$$

Let $\bar{H}$ be the Hugoniot curve with center $\left(\tau_{2}, \bar{p}\right)$, and let $\tau=\bar{V}(p)$ along $\bar{H}$ for $p \in(0, \bar{p}]$, as in Lemma 2.18. By (4.23),

$$
\left(-g_{\tau}\left(\tau_{2}, \bar{p}\right)\right) \frac{\partial}{\partial p} e\left(\tau_{2}, \bar{p}\right)-\frac{\bar{p}}{2}<0 ;
$$

hence we can find $p_{0} \in(0, \bar{p})$ such that

$$
\left(-g_{\tau}\left(\tau_{2}, \bar{p}\right)\right) \frac{\partial}{\partial p} e\left(\tau_{2}, \bar{p}\right)-\frac{\bar{p}-p_{0}}{2}<0 .
$$

Write $\tau_{0}=\bar{V}\left(p_{0}\right)$; then $\left(\tau_{0}, p_{0}\right) \in \bar{H}$, so $\left(\tau_{2}, \bar{p}\right) \in H$. But (4.19) holds for any $(\tau, p) \in H$ for which $H_{\tau}(\tau, p) \neq 0$ and $p>p_{0}$; in particular (4.19) holds for $(\tau, p)=\left(\tau_{2}, \bar{p}\right)$, by (4.24) and (2.2). Then by (4.24) we see that 


$$
\begin{aligned}
\left.\frac{d \theta}{d p}\right|_{\left(\tau_{2}, \bar{p}\right)} & <0 \Leftrightarrow\left[\left(-g_{\tau}\left(\tau_{2}, \bar{p}\right)\right) \frac{\partial}{\partial p} e\left(\tau_{2}, \bar{p}\right)-\frac{\bar{p}-p_{0}}{2}\right]\left(\tau_{0}-\tau_{2}\right) \\
& >\left(\bar{p}-p_{0}\right)\left[\frac{\partial}{\partial p} e\left(\tau_{2}, \bar{p}\right)-\frac{\tau_{0}-\tau_{2}}{2}\right] \\
& \Leftrightarrow \frac{\partial}{\partial p} e\left(\tau_{2}, \bar{p}\right)\left[\left(-g_{\tau}\left(\tau_{2}, \bar{p}\right)\right)\left(\tau_{0}-\tau_{2}\right)-\left(\bar{p}-p_{0}\right)\right]>0
\end{aligned}
$$

which is true by Corollary 2.4 and (1.22). Then also

$$
d p /\left.d \theta\right|_{\left(\tau_{2}, \bar{p}\right)}<0 \text {. }
$$

It follows from (4.22) that

$$
d \tau /\left.d p\right|_{\left(\tau_{2}, \bar{p}\right)}<0 .
$$

Combining Lemmas 4.10 and 4.11 gives

LEMMA 4.12. Suppose e violates MEDIUM. Then there exist $\left(\tau_{0}, p_{0}\right)$, and $\left(\tau_{1}\right.$, $\left.p_{1}\right) \in H \cap Q$, such that

$$
\left.\frac{d a}{d \theta}\right|_{\left(\tau_{1}, p_{1}\right)}<0,\left.\quad \frac{d p}{d \theta}\right|_{\left(\tau_{1}, p_{1}\right)} \neq 0
$$

THEOREM 4.13. Suppose e violates MEDIUM. Then uniqueness fails; there exist initial data $\left(U_{l}, U_{r}\right)$ for which 3-fold type I nonuniqueness holds.

Proof. By Lemma 4.12, there exist $\left(\tau_{0}, p_{0}\right)$, and $\left(\tau_{2}, p_{2}\right) \in H \cap Q$, such that

$$
d a /\left.d \theta\right|_{\left(\tau_{2}, p_{2}\right)}<0 .
$$

Let $\theta_{2}=\theta\left(\tau_{2}, p_{2}\right)$ (and, as always, $\left.\theta_{0}=\theta\left(\tau_{0}, p_{0}\right)=-d p /\left.d \tau\right|_{\left(\tau_{0}, p_{0}\right)}\right)$. Then

$$
d a^{0}(\theta) /\left.d \theta\right|_{\theta=\theta_{2}}<0 \text {. }
$$

Thus $a^{0}(\theta)>a^{0}\left(\theta_{2}\right)$ for $\theta<\theta_{2}$, $\theta$ near $\theta_{2}$; $a^{0}(\theta)<a^{0}\left(\theta_{2}\right)$ for $\theta>\theta_{2}$, near $\theta_{2}$. But $a^{0}\left(\theta_{0}\right)=0$, so $\exists \theta_{1} \in\left(\theta_{0}, \theta_{2}\right)$ such that $a^{0}\left(\theta_{1}\right)=a^{0}\left(\theta_{2}\right)$. Moreover, by Lemma 2.22 , we can find arbitrarily large $\theta$ such that $a^{0}(\theta)>a^{0}\left(\theta_{2}\right)$, so $\exists \theta_{3} \in\left(\theta_{2},+\infty\right)$ such that $a^{0}\left(\theta_{3}\right)=a^{0}\left(\theta_{2}\right)$. Let $a=a^{0}\left(\theta_{2}\right)$, and let $\left(\tau_{i}\right.$, $\left.p_{i}\right)=\left(\tau^{0}\left(\theta_{i}\right), p^{0}\left(\theta_{i}\right)\right)$ for $i=1,2,3$. Then the $\left(\tau_{i}, p_{i}\right)$ 's are distinct and satisfy (4.11). Let $U_{l}=\left(\tau_{0}, p_{0}, u_{l}\right), U_{r}=\left(\tau_{0}, p_{0}, u_{l}-2 a\right)$, where $u_{l}$ is arbitrary. Then 3-fold type I nonuniqueness holds for $\left(U_{l}, U_{r}\right)$. (See Figure 11.)

Thus we see from Theorem 4.5 and Theorem 4.13 that the condition MEDIUM is necessary and sufficient for uniqueness of solutions of the R. P.

We conclude this section by showing that when WEAK fails, type II nonuniqueness occurs.

LEMMA 4.14. Suppose e violates WEAK. Then there exist a point $\left(\tau_{0}, p_{0}\right)$, an open interval $N \subset\left(p_{0},+\infty\right)$ and three $C^{1}$ functions $V_{1}, V_{2}, V_{3}$ on $N$ such that 


$$
\begin{gathered}
\left(V_{i}(p), p\right) \in H \quad(p \in N, i=1,2,3), \\
V_{1}(p)<V_{2}(p)<V_{3}(p) \quad(p \in N), \\
V_{2}^{\prime}(p)<0 \quad(p \in N), \\
H_{\tau}\left(V_{i}(p), p\right) \neq 0 \quad(p \in N, i=1,2,3) .
\end{gathered}
$$

(See Figure 12.)

Proof. Use Lemma 4.11.

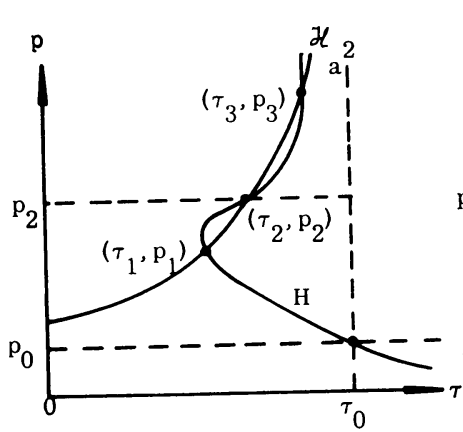

WEAK and not-MEDIUM

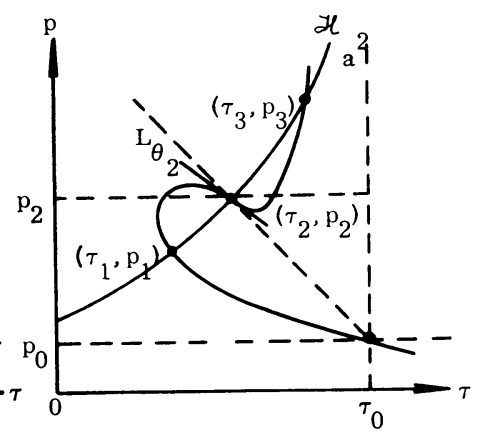

not-WEAK

FIGURE 11. 3-fold type I nonuniqueness occurs whenever MEDIUM fails.

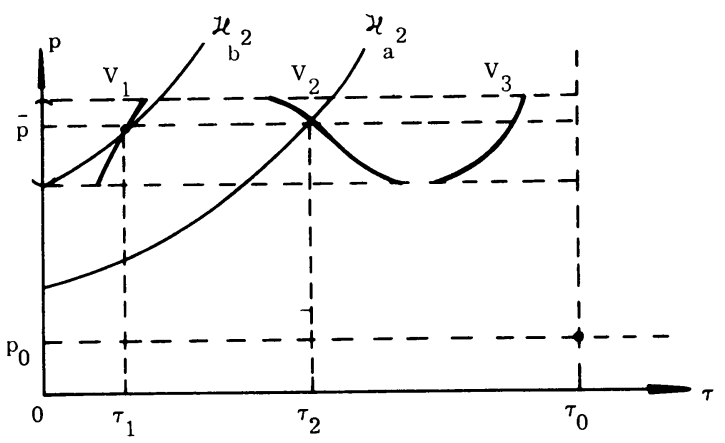

Figure 12. Type II nonuniqueness.

THEOREM 4.15. Suppose e violates WEAK. Then there exist initial data ( $U_{l}$, $\left.U_{r}\right)$ for which type II nonuniqueness holds.

Proof. Let $\left(\tau_{0}, p_{0}\right), N, V_{1}, V_{2}$ and $V_{3}$ be as in Lemma 4.14. Choose $\bar{p} \in N$, and let $\tau_{1}=V_{1}(\bar{p}), \tau_{2}=V_{2}(\bar{p})$; then $\left(\tau_{1}, \bar{p}\right),\left(\tau_{2}, \bar{p}\right) \in H$. Also, $\bar{p}>p_{0}$, so by Corollary 2.7, $\tau_{1}, \tau_{2}<\tau_{0}$; by (4.26), $\tau_{1}<\tau_{2}$. (See Figure 12.) It is now clear from Definition 4.8 and the remarks preceding it that data $\left(U_{l}, U_{r}\right)$ can be found for which type II nonuniqueness holds. 
Observe that, although the violation of WEAK implies the occurrence of both type I and type II nonuniqueness, the data $\left(U_{l}, U_{r}\right)$ given by Theorems 4.13 and 4.15 need not be the same. In the next section we shall prove that (when WEAK fails) there always exists one set of data $\left(U_{l}, U_{r}\right)$ for which type I and type II nonuniqueness hold simultaneously, and for which the resulting 5 -fold nonuniqueness is stable.

5. Stability of the nonunique solutions. We begin by providing a meaning for the title of this section.

Suppose we have initial data $\left(U_{l}^{0}, U_{r}^{0}\right)$ for which $n$-fold nonuniqueness (type I, type II, both, or neither) holds, i.e., the R. P. with data $\left(U_{l}^{0}, U_{r}^{0}\right)$ has $n$ distinct solutions,

$$
U_{l}^{0} \stackrel{1}{-} U_{1}^{i, 0} \stackrel{\text { contact }}{U_{3}^{i, 0}} \stackrel{3}{-} U_{r}^{0} \quad(1 \leqslant i \leqslant n) .
$$

Definition 5.1. We say the $n$-fold nonuniqueness is stable if there exists a neighborhood $\Re$ of $\left(U_{l}^{0}, U_{r}^{9}\right)$ such that

(a) for any $\left(U_{l}, U_{r}\right) \in \Re$, the R. P. with data $\left(U_{l}, U_{r}\right)$ has $n$ solutions;

(b) these $n$ solutions can be expressed,

$$
U_{l} \stackrel{1}{-} U_{1}^{i}\left(U_{l}, U_{r}\right) \stackrel{\text { contact }}{=} U_{3}^{i}\left(U_{l}, U_{r}\right) \stackrel{3}{-} U_{r} \quad\left(\left(U_{l}, U_{r}\right) \in, i=1, \ldots, n\right),
$$

in such a way that

$$
U_{j}^{i}\left(U_{l}, U_{r}\right) \rightarrow U_{j}^{i, 0} \quad(j=1,3 ; i=1, \ldots, n)
$$

as $\left(U_{l}, U_{r}\right) \rightarrow\left(U_{l}^{0}, U_{r}^{0}\right)$.

In this section we shall prove that for all types of $n$-fold nonuniqueness considered, the data can be chosen so that the $n$-fold nonuniqueness is stable. Thus when MEDIUM fails, there exist data that exhibit 3-fold type I nonuniqueness, and for which the 3-fold nonuniqueness is stable. (See Theorem 5.4.) When WEAK fails, we can find data that exhibit simultaneously 3-fold type I nonuniqueness and (2-fold) type II nonuniqueness, and for which the 5-fold nonuniqueness is stable. (See Theorem 5.6.)

We begin with type I nonuniqueness; the following two lemmas provide sufficient conditions for stability.

Lemma 5.2. Suppose $\theta_{0}<\theta_{1}<\theta_{2}<\theta_{3}, a>0$ and

$$
\begin{aligned}
& d a /\left.d \theta\right|_{\theta=\theta_{i}} \neq 0 \quad(i=1,2,3), \\
& d p /\left.d \theta\right|_{\theta=\theta_{i}} \neq 0 \quad(i=1,2,3), \\
& a^{0}\left(\theta_{i}\right)=a \quad(i=1,2,3) .
\end{aligned}
$$

Let $u_{0}$ be arbitrary and let $U_{l}^{0}=\left(\tau_{0}, p_{0}, u_{0}\right), U_{r}^{0}=\left(\tau_{0}, p_{0}, u_{0}-2 a\right)$. Then the $R$. $P$. with data $\left(U_{l}^{0}, U_{r}^{0}\right)$ has three type I solutions for which the 3-fold nonuniqueness is stable. 
Proof. Let $\left(\tau_{i}, p_{i}\right)=\left(\tau^{0}\left(\theta_{i}\right), p^{0}\left(\theta_{i}\right)\right)(i=1,2,3)$. Then by (5.4), the R. P. with data $\left(U_{l}^{0}, U_{r}^{0}\right)$ has the three type I solutions,

$$
\begin{aligned}
& U_{l}^{0} \stackrel{\text { 1-shock }}{ }\left(\tau_{i}, p_{i}, u_{0}-a\right) \stackrel{\text { contact }}{\frac{\text { 3-shock }}{}}\left(\tau_{i}, p_{i}, u_{0}-a\right) \stackrel{0}{ } \\
& (i=1,2,3) \text {; }
\end{aligned}
$$

which we have expressed in the form of (5.1). From (5.3), it is easy to see that $H_{\tau}\left(\tau_{i}, p_{i}\right) \neq 0(i=1,2,3)$. For any point $\left(\tau_{c}, p_{c}\right)$ as center, we define

$$
H\left(\tau_{c}, p_{c} ; \tau, p\right)=e(\tau, p)-e\left(\tau_{c}, p_{c}\right)+\left(\tau-\tau_{c}\right) \frac{p+p_{c}}{2} \quad\left(\tau_{c}, p_{c}, \tau, p>0\right) .
$$

Then $H_{\tau}\left(\tau_{0}, p_{0} ; \tau_{i}, p_{i}\right) \neq 0(i=1,2,3)$, so by the implicit function theorem there exist neighborhoods $N_{i}$ of $\left(\tau_{0}, p_{0}, p_{i}\right)$ and $C^{1}$ functions $V_{i}$ on $N_{i}$ such that

$$
\begin{aligned}
V_{i}\left(\tau_{0}, p_{0} ; p_{i}\right) & =\tau_{i} \quad(i=1,2,3), \\
H\left(\tau_{c}, p_{c} ; V_{i}\left(\tau_{c}, p_{c} ; p\right), p\right) & =0 \quad\left(\left(\tau_{c}, p_{c}, p\right) \in N_{i}, i=1,2,3\right),
\end{aligned}
$$

and we may assume

$$
\begin{gathered}
N_{i} \cap N_{j}=\varnothing \quad(1 \leqslant i<j \leqslant 3), \\
\left(\tau_{c}, p_{c}, p\right) \in N_{i} \Rightarrow p>p_{c} \quad(i=1,2,3) .
\end{gathered}
$$

For brevity, we shall usually write

$$
V_{i}^{c}(p)=V_{i}\left(\tau_{c}, p_{c} ; p\right) \quad\left(\left(\tau_{c}, p_{c}, p\right) \in N_{i}\right) .
$$

We also define

$$
\eta\left(\tau_{c}, p_{c} ; \tau, p\right)=\sqrt{\left(\tau_{c}-\tau\right)\left(p-p_{c}\right)},
$$

and we abbreviate $\eta^{c}(\tau, p)=\eta\left(\tau_{c}, p_{c} ; \tau, p\right)$. In similar fashion, we define

$$
a_{c}(p)=\eta^{c}\left(V_{i}^{c}(p), p\right) \text { when }\left(\tau_{c}, p_{c}, p\right) \in N_{i} .
$$

By Lemma 2.9, (5.2), and the fact that $H_{\tau}\left(\tau_{i}, p_{i}\right) \neq 0$, we have

$$
\left.\frac{d a}{d p}\right|_{\left(\tau_{i}, p_{i}\right)}=\left.\left.\frac{d a}{d \theta}\right|_{\left(\tau_{i}, p_{i}\right)} \cdot \frac{d \theta}{d p}\right|_{\left(\tau_{i}, p_{i}\right)} \neq 0 \quad(i=1,2,3),
$$

which, in the notation (5.10)-(5.12), can be expressed,

$$
a_{0}^{\prime}\left(p_{i}\right) \neq 0 \quad(i=1,2,3) .
$$

Define

$$
\Phi\left(\tau_{l}, p_{l}, u_{l}, \tau_{r}, p_{r}, p\right)=\left(\tau_{l}, p_{l}, u_{l}, \tau_{r}, p_{r}, u_{l}-\left(a_{l}(p)+a_{r}(p)\right)\right),
$$

for all $\left(\tau_{l}, p_{l}, u_{l}, \tau_{r}, p_{r}, p\right)$ for which $\left(\tau_{l}, p_{l}, p\right),\left(\tau_{r}, p_{r}, p\right) \in N_{i}$ for some $i$. (Here, for example, $a_{l}(p)=\eta^{l}\left(V_{i}^{l}(p), p\right)$ when $\left(\tau_{l}, p_{l}, p\right) \in N_{i}$; $\operatorname{see}(5.10)-(5.12)$.) $\Phi$ is 
a $C^{1}$ function defined on an open set containing $\left\{\left(\tau_{0}, p_{0}, u_{0}, \tau_{0}, p_{0}, p_{i}\right) \mid i=1\right.$, $2,3\}$, and has Jacobian matrix of the form

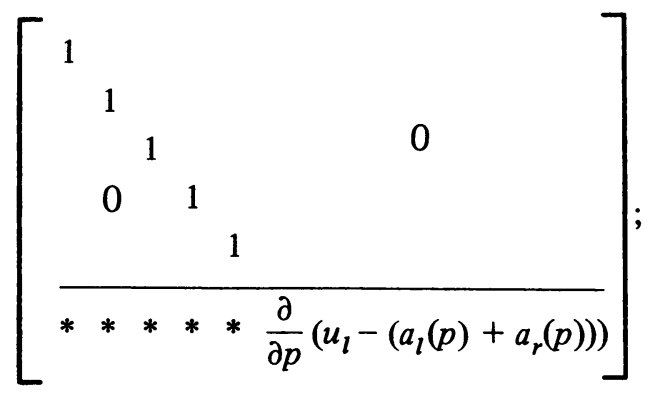

hence its Jacobian determinant is given by

$$
J_{\Phi}\left(\tau_{l}, p_{l}, u_{l}, \tau_{r}, p_{r}, p\right)=\frac{\partial}{\partial p}\left(u_{l}-\left(a_{l}(p)+a_{r}(p)\right)\right)=-\left(a_{l}^{\prime}(p)+a_{r}^{\prime}(p)\right),
$$

whence

$$
J_{\Phi}\left(\tau_{0}, p_{0}, u_{0}, \tau_{0}, p_{0}, p_{i}\right)=-\left(a_{0}^{\prime}\left(p_{i}\right)+a_{0}^{\prime}\left(p_{i}\right)\right)=-2 a_{0}^{\prime}\left(p_{i}\right) \neq 0
$$

$$
(i=1,2,3) \text {. }
$$

Moreover,

$$
\begin{aligned}
\Phi\left(\tau_{0}, p_{0}, u_{0}, \tau_{0}, p_{0}, p_{i}\right) & =\left(\tau_{0}, p_{0}, u_{0}, \tau_{0}, p_{0}, u_{0}-\left(a_{0}\left(p_{i}\right)+a_{0}\left(p_{i}\right)\right)\right) \\
& =\left(\tau_{0}, p_{0}, u_{0}, \tau_{0}, p_{0}, u_{0}-2 a\right) \quad(i=1,2,3),
\end{aligned}
$$

since $a_{0}\left(p_{i}\right)=\eta^{0}\left(\tau_{i}, p_{i}\right)=a^{0}\left(\theta_{i}\right)=a$ by (5.4) and (5.7). Hence there exist neighborhoods $\Re_{i}$ of $\left(\tau_{0}, p_{0}, u_{0}, \tau_{0}, p_{0}, p_{i}\right)$ and $\Re_{i}$ of $\left(\tau_{0}, p_{0}, u_{0}, \tau_{0}, p_{0}\right.$ $\left.u_{0}-2 a\right)$ such that $\left.\Phi\right|_{\mathscr{K}_{i}}$ is a $C^{1}$ diffeomorphism of $\Re_{i}$ onto $\Re_{i}$; we may assume

$$
\mathfrak{K}_{i} \cap \mathfrak{M}_{j}=\varnothing \quad(1<i<j<3) .
$$

In addition, (5.9) implies

$$
\left(\tau_{l}, p_{l}, u_{l}, \tau_{r}, p_{r}, p\right) \in \mathfrak{N}_{i} \Rightarrow p>p_{l}, p_{r} \quad(i=1,2,3) .
$$

Observe that $\left(U_{l}^{0}, U_{r}^{0}\right)=\left(\tau_{0}, p_{0}, u_{0}, \tau_{0}, p_{0}, u_{0}-2 a\right)$. Let $\Re=\Re_{1} \cap \Re_{2} \cap$ $\Re_{3} ; \Re$ is a neighborhood of $\left(U_{l}^{0}, U_{r}^{g}\right)$. Let $\left(U_{l}, U_{r}\right) \in \Re$. Then $\forall i,\left(\tau_{l}, p_{l}, u_{l}\right.$, $\left.\tau_{r}, p_{r}, u_{r}\right)=\left(U_{l}, U_{r}\right) \in \Re_{i}$, so there is a $\bar{p}_{i}>0$ with $\left(\tau_{l}, p_{l}, u_{l}, \tau_{r}, p_{r}, \bar{p}_{i}\right) \in \Re_{i}$ such that $\Phi\left(\tau_{l}, p_{l}, u_{l}, \tau_{r}, p_{r}, \bar{p}_{i}\right)=\left(\tau_{l}, p_{l}, u_{l}, \tau_{r}, p_{r}, u_{r}\right)$. Since also $\Phi\left(\tau_{l}, p_{l}, u_{l}, \tau_{r}\right.$, $\left.p_{r}, \overline{p_{i}}\right)=\left(\tau_{l}, p_{l}, u_{l}, \tau_{r}, p_{r}, u_{l}-\left(a_{l}\left(\overline{p_{i}}\right)+a_{r}\left(\overline{p_{i}}\right)\right)\right)$, we have

$$
u_{r}=u_{l}-\left(a_{l}\left(\bar{p}_{i}\right)+a_{r}\left(\bar{p}_{i}\right)\right) \quad(i=1,2,3) ;
$$

moreover $\bar{p}_{i}>p_{l}, p_{r}$ by (5.16). Here $\bar{p}_{i}$ is a $C^{1}$ function of $\left(U_{l}, U_{r}\right)$; in fact, it is the last coordinate function of the $C^{1}$ diffeomorphism $\left.\left(\left.\Phi\right|_{\mathscr{N}_{i}}\right)^{-1}\right|_{\mathscr{r}}$. Note that 
by (5.15), the $\bar{p}_{i}$ 's are distinct. Thus from (5.8) and (5.17) we see that the R. P. with data $\left(U_{l}, U_{r}\right)$ has the following three distinct solutions, for $i=1,2,3$ :

$$
\begin{aligned}
& U_{l} \stackrel{\text { 1-shock }}{ }\left(V_{i}^{l}\left(\overline{p_{i}}\right), \overline{p_{i}}, u_{l}-a_{l}\left(\overline{p_{i}}\right)\right) \\
& \stackrel{\text { contact }}{\longrightarrow}\left(V_{i}^{r}\left(\overline{p_{i}}\right), \overline{p_{i}}, u_{r}+a_{r}\left(\overline{p_{i}}\right)\right) \stackrel{\text { 3-shock }}{=} U_{r},
\end{aligned}
$$

which establishes condition (a) of Definition 5.1.

By (5.14) and the fact that $\left(\left.\Phi\right|_{\Re_{i}}\right)^{-1}$ is continuous on $\Re_{i}, \bar{p}_{i} \rightarrow p_{i}$ as $\left(U_{l}\right.$, $\left.U_{r}\right) \rightarrow\left(U_{l}^{0}, U_{r}^{0}\right)$. Letting

$$
\begin{aligned}
& U_{1}^{i}\left(U_{l}, U_{r}\right)=\left(V_{i}^{l}\left(\overline{p_{i}}\right), \overline{p_{i}}, u_{l}-a_{l}\left(\overline{p_{i}}\right)\right), \\
& U_{3}^{i}\left(U_{l}, U_{r}\right)=\left(V_{i}^{r}\left(\overline{p_{i}}\right), \overline{p_{i}}, u_{r}+a_{r}\left(\overline{p_{i}}\right)\right),
\end{aligned}
$$

we see that the solutions (5.18) are expressible as

$$
U_{l} \stackrel{1}{-} U_{1}^{i}\left(U_{l}, U_{r}\right) \stackrel{\text { contact }}{ } U_{3}^{i}\left(U_{l}, U_{r}\right) \stackrel{3}{-} U_{r} \quad(i=1,2,3),
$$

and $U_{j}^{i}\left(U_{l}, U_{r}\right) \rightarrow\left(\tau_{i}, p_{i}, u_{0}-a\right)$ as $\left(U_{l}, U_{r}\right) \rightarrow\left(U_{l}^{0}, U_{r}^{0}\right)(i=1,2,3 ; j=1,3)$ (see (5.17)). Referring to (5.1) and (5.5), we see that condition (b) of Definition 5.1 holds.

We can also establish

LEMMA 5.3. Let $\theta_{0}<\overline{\theta_{1}}<\bar{\theta}<\overline{\theta_{2}}$, and suppose $a^{0}\left(\overline{\theta_{1}}\right)>a^{0}(\bar{\theta})>a^{0}\left(\overline{\theta_{2}}\right)$, $\left(a^{0}\right)^{\prime}(\bar{\theta}) \neq 0$ and $\left(p^{0}\right)^{\prime}(\bar{\theta}) \neq 0$. Then for any open interval I containing $a^{0}(\bar{\theta})$, there exist $\theta_{1}, \theta_{2}, \theta_{3}$ and $a \in I$ with $\theta_{0}<\theta_{1}<\theta_{2}<\theta_{3}$, such that $a^{0}\left(\theta_{i}\right)=a$, $\left(a^{0}\right)^{\prime}\left(\theta_{i}\right) \neq 0$ and $\left(p^{0}\right)^{\prime}\left(\theta_{i}\right) \neq 0(i=1,2,3)$.

As a corollary to the preceding two lemmas and Lemma 4.12, we have

THEOREM 5.4. Suppose e violates MEDIUM. Then there exist data $\left(U_{l}^{0}, U_{r}^{0}\right)$ for which 3-fold type I nonuniqueness holds and the 3-fold nonuniqueness is stable.

Proof. By Lemma 4.12 , there exist a center $\left(\tau_{0}, p_{0}\right)$, and a point $(\bar{\tau}$, $\bar{p}) \in H \cap Q$ (see (2.1)), such that

$$
\left.\frac{d a}{d \theta}\right|_{(\bar{\tau}, \bar{p})}<0,\left.\quad \frac{d p}{d \theta}\right|_{(\bar{\tau}, \bar{p})} \neq 0 .
$$

Let $\bar{\theta}=\theta(\bar{\tau}, \bar{p})$; then $\bar{\theta}>\theta_{0},\left(a^{0}\right)^{\prime}(\bar{\theta})<0$ and $\left(p^{0}\right)^{\prime}(\bar{\theta}) \neq 0$. Then there exist $\overline{\theta_{1}} \in\left(\theta_{0}, \bar{\theta}\right)$ and $\overline{\theta_{2}}>\bar{\theta}$ such that $a^{0}\left(\overline{\theta_{1}}\right)>a^{0}(\bar{\theta})>a^{0}\left(\overline{\theta_{2}}\right)$. By Lemma 5.3, there exist $\theta_{1}, \theta_{2}, \theta_{3}$ and $a>0$ with $\theta_{0}<\theta_{1}<\theta_{2}<\theta_{3}$, such that $a^{0}\left(\theta_{i}\right)=a$, $\left(a^{0}\right)^{\prime}\left(\theta_{i}\right) \neq 0$ and $\left(p^{0}\right)^{\prime}\left(\theta_{i}\right) \neq 0(i=1,2,3)$. Let $u_{0}$ be arbitrary and let $U_{l}^{0}=\left(\tau_{0}, p_{0}, u_{0}\right), U_{r}^{0}=\left(\tau_{0}, p_{0}, u_{0}-2 a\right)$. By Lemma 5.2 the R. P. with data $\left(U_{l}^{0}, U_{r}^{0}\right)$ has three type $\mathrm{I}$ solutions for which the 3 -fold nonuniqueness is stable. 
In preparation for Theorem 5.6, we prove the following lemma, which gives a sufficient condition for stability in the case of type II nonuniqueness.

Lemma 5.5. Suppose $\left(\tau_{1}, \bar{p}\right),\left(\tau_{2}, \vec{p}\right) \in H \cap Q$ with $\tau_{1}<\tau_{2}$ and

$$
\begin{aligned}
& H_{\tau}\left(\tau_{1}, \bar{p}\right), H_{\tau}\left(\tau_{2}, \bar{p}\right) \neq 0, \\
& \left.\frac{d a}{d p}\right|_{\left(\tau_{1}, \bar{p}\right)}+\left.\frac{d a}{d p}\right|_{\left(\tau_{2}, \bar{p}\right)} \neq 0 .
\end{aligned}
$$

Let $u_{0}$ be arbitrary and let $U_{l}^{0}=\left(\tau_{0}, p_{0}, u_{0}\right), U_{r}^{0}=\left(\tau_{0}, p_{0}, u_{0}-\left(a_{0}+b_{0}\right)\right)$, where $a_{0}=\eta^{0}\left(\tau_{2}, \bar{p}\right), b_{0}=\eta^{0}\left(\tau_{1}, \bar{p}\right)$. Then type II nonuniqueness holds for the data $\left(U_{l}^{0}, U_{r}^{0}\right)$, and the (2-fold) nonuniqueness is stable.

Proof. First recall that the R. P. with data $\left(U_{l}^{0}, U_{r}^{0}\right)$ has the two type II solutions,

$$
\begin{aligned}
& U_{l}^{0} \stackrel{\text { 1-shock }}{ }\left(\tau_{1}, \bar{p}, u_{l}-b_{0}\right) \stackrel{\text { contact }}{ }\left(\tau_{2}, \bar{p}, u_{l}-b_{0}\right) \stackrel{\text { 3-shock }}{{ }^{3}} U_{r}^{0}, \\
& U_{l}^{0} \stackrel{\text { 1-shock }}{=}\left(\tau_{2}, \bar{p}, u_{l}-a_{0}\right) \stackrel{\text { contact }}{=}\left(\tau_{1}, \bar{p}, u_{l}-a_{0}\right) \stackrel{\text { 3-shock }}{=} U_{r}^{0} \text {. }
\end{aligned}
$$

By (5.19), $H_{\tau}\left(\tau_{0}, p_{0} ; \tau_{1}, \bar{p}\right), H_{\tau}\left(\tau_{0}, p_{0} ; \tau_{2}, \bar{p}\right) \neq 0$ (see (5.6)); hence we can find a neighborhood $N$ of $\left(\tau_{0}, p_{0}, \bar{p}\right)$ and $C^{1}$ functions $V_{1}$ and $V_{2}$ on $N$ such that, abbreviating $V_{i}\left(\tau_{c}, p_{c} ; p\right)=V_{i}^{c}(p)$, we have

$$
\begin{gathered}
V_{i}^{0}(\bar{p})=\tau_{i} \quad(i=1,2), \\
H\left(\tau_{c}, p_{c} ; V_{i}^{c}(p), p\right)=0 \quad\left(\left(\tau_{c}, p_{c}, p\right) \in N, i=1,2\right), \\
\left(\tau_{c}, p_{c}, p\right) \in N \Rightarrow p>p_{c}, \\
\left(\tau_{c}, p_{c}, p\right),\left(\tau_{d}, p_{d}, q\right) \in N \Rightarrow V_{1}^{c}(p)<V_{2}^{d}(q) .
\end{gathered}
$$

Let $\mathfrak{V}=\left\{\left(\tau_{l}, p_{l}, \tau_{r}, p_{r}, p, q\right) \mid\left(\tau_{l}, p_{l}, p\right),\left(\tau_{l}, p_{l}, q\right),\left(\tau_{r}, p_{r}, p\right),\left(\tau_{r}, p_{r}, q\right) \in N\right\} ; \mathcal{V}$ is an open set containing $\left(\tau_{0}, p_{0}, \tau_{0}, p_{0}, \bar{p}, \bar{p}\right)$. For $\left(\tau_{l}, p_{l}, \tau_{r}, p_{r}, p, q\right) \in \mathcal{V}$, we define

$$
\begin{array}{ll}
a_{r}=\eta^{r}\left(V_{2}^{r}(p), p\right), & a_{l}=\eta^{l}\left(V_{2}^{l}(q), q\right), \\
b_{r}=\eta^{r}\left(V_{1}^{r}(q), q\right), & b_{l}=\eta^{l}\left(V_{1}^{l}(p), p\right) .
\end{array}
$$

(See Figure 13; $\mathcal{H}_{a}^{c}$ is the upper branch of the hyperbola $\left(\tau_{c}-\tau\right)\left(p-p_{c}\right)=$ a.) Suppose $u_{l}$ and $u_{r}$ satisfy

$$
u_{r}=u_{l}-\left(a_{r}+b_{l}\right)
$$

then by (5.23) and (5.24), the R. P. with data $\left(U_{l}, U_{r}\right)=\left(\tau_{l}, p_{l}, u_{l}, \tau_{r}, p_{r}, u_{r}\right)$ has the solution,

$$
U_{l} \stackrel{\text { 1-shock }}{ }\left(V_{1}^{l}(p), p, u_{l}-b_{l}\right) \stackrel{\text { contact }}{ }\left(V_{2}^{r}(p), p, u_{r}+a_{r}\right) \stackrel{\text { 3-shock }}{=} U_{r},
$$




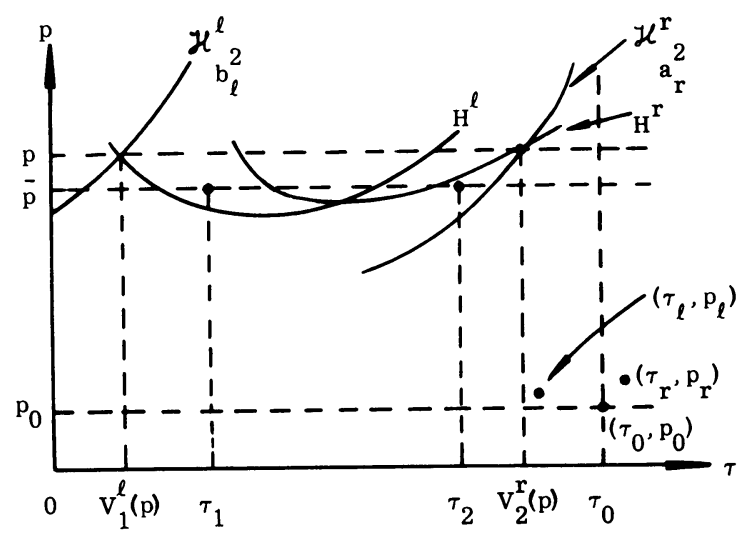

Figure 13. One of the solutions.

since $u_{l}-b_{l}=u_{r}+a_{r}$ by (5.26). (See Figure 13.) If, on the other hand, $u_{l}$ and $u_{r}$ satisfy

$$
u_{r}=u_{l}-\left(a_{l}+b_{r}\right),
$$

then $u_{l}-a_{l}=u_{r}+b_{r}$, and the R. P. with data $\left(U_{l}, U_{r}\right)$ has the solution,

$$
U_{l} \stackrel{\text { 1-shock }}{ }\left(V_{2}^{l}(q), q, u_{l}-a_{l}\right) \stackrel{\text { contact }}{ }\left(V_{1}^{r}(q), q, u_{r}+b_{r}\right) \stackrel{\text { 3-shock }}{=} U_{r} \text {. }
$$

Thus if $u_{l}$ and $u_{r}$ satisfy (5.26) and (5.28) simultaneously, then the R. P. with data $\left(U_{l}, U_{r}\right)$ has the two solutions (5.27) and (5.29), which are distinct by (5.25). But (5.26) and (5.28) hold iff

$$
a_{l}+b_{r}=a_{r}+b_{l} \text {. }
$$

Define $\Phi\left(\tau_{l}, p_{l}, \tau_{r}, p_{r}, p, q\right)=a_{l}+b_{r}-a_{r}-b_{l}\left(\left(\tau_{l}, p_{l}, \tau_{r}, p_{r}, p, q\right) \in \mathcal{V}\right)$. (Note that, for example, $a_{l}$ is defined as a number depending on the variables $\tau_{l}, p_{l}, q$.) Then $\Phi\left(\tau_{0}, p_{0}, \tau_{0}, p_{0}, \bar{p}, \bar{p}\right)=0$ (since, for example, $a_{l}\left(\tau_{0}, p_{0}, \tau_{0}, p_{0}, \bar{p}\right.$, $\left.\bar{p})=\eta^{0}\left(V_{2}^{0}(\bar{p}), \bar{p}\right)=\eta^{0}\left(\tau_{2}, \bar{p}\right)=a_{0}\right)$. Moreover,

$$
\frac{\partial}{\partial q} \Phi\left(\tau_{l}, p_{l}, \tau_{r}, p_{r}, p, q\right)=\frac{d}{d q} \eta^{l}\left(V_{2}^{l}(q), q\right)+\frac{d}{d q} \eta^{r}\left(V_{1}^{r}(q), q\right) ;
$$

then

$$
\frac{\partial \Phi}{\partial q}\left(\tau_{0}, p_{0}, \tau_{0}, p_{0}, \bar{p}, \bar{p}\right)=\left.\frac{d a}{d p}\right|_{\left(\tau_{2}, \bar{p}\right)}+\left.\frac{d a}{d p}\right|_{\left(\tau_{1}, \bar{p}\right)} \neq 0,
$$

by (5.6), (5.22), (5.23), Conventions 4.6 and 4.9, and (5.20). Thus by the implicit function theorem, there exist a neighborhood W of $\left(\tau_{0}, p_{0}, \tau_{0}, p_{0}, \vec{p}\right)$ and a $C^{1}$ function $q=q\left(\tau_{l}, p_{l}, \tau_{r}, p_{r}, p\right)\left(\left(\tau_{l}, p_{l}, \tau_{r}, p_{r}, p\right) \in \mathcal{W}\right)$ such that $q\left(\tau_{0}\right.$ $\left.p_{0}, \tau_{0}, p_{0}, \bar{p}\right)=\bar{p}$ and

$$
\Phi\left(\tau_{l}, p_{l}, \tau_{r}, p_{r}, p, q\left(\tau_{l}, p_{l}, \tau_{r}, p_{r}, p\right)\right)=0 \quad\left(\left(\tau_{l}, p_{l}, \tau_{r}, p_{r}, p\right) \in \mathscr{W}\right) .
$$


Henceforth we shall abbreviate $q\left(\tau_{l}, p_{l}, \tau_{r}, p_{r}, p\right)=q$. Thus $q$ is a number depending continuously on the variables $\tau_{l}, p_{l}, \tau_{r}, p_{r}$ and $p$. Using this $q$ in the definitions of $a_{l}$ and $b_{r}$ above, we see that $a_{r}, b_{r}, a_{l}$ and $b_{l}$ likewise are numbers depending continuously on the variables $\tau_{l}, p_{l}, \tau_{r}, p_{r}$ and $p$. Here $q$, $a_{r}, b_{r}, a_{l}$ and $b_{l}$ are defined whenever $\left(\tau_{l}, p_{l}, \tau_{r}, p_{r}, p\right) \in$ W. Thus (5.31) becomes

$$
a_{l}+b_{r}=a_{r}+b_{l} \quad\left(\left(\tau_{l}, p_{l}, \tau_{r}, p_{r}, p\right) \in \mathcal{W}\right) .
$$

Now for $\left(\tau_{l}, p_{l}, \tau_{r}, p_{r}, p\right) \in \mathcal{W}$ and $u_{l}$ arbitrary, we define

$$
\Psi\left(\tau_{l}, p_{l}, u_{l}, \tau_{r}, p_{r}, p\right)=\left(\tau_{l}, p_{l}, u_{l}, \tau_{r}, p_{r}, u_{l}-\left(a_{r}+b_{l}\right)\right) .
$$

Clearly $\Psi$ has Jacobian determinant given by

$$
\begin{aligned}
& J_{\Psi}\left(\tau_{l}, p_{l}, u_{l}, \tau_{r}, p_{r}, p\right)= \frac{\partial}{\partial p}\left(u_{l}-\left(a_{r}+b_{l}\right)\right) \\
&=-\left(\frac{d}{d p} \eta^{r}\left(V_{2}^{r}(p), p\right)+\frac{d}{d p} \eta^{l}\left(V_{1}^{l}(p), p\right)\right) \\
& \quad\left(\left(\tau_{l}, p_{l}, \tau_{r}, p_{r}, p\right) \in W\right) .
\end{aligned}
$$

Then clearly $-J_{\Psi}\left(\tau_{0}, p_{0}, u_{0}, \tau_{0}, p_{0}, \vec{p}\right) \neq 0$; moreover

$$
\Psi\left(\tau_{0}, p_{0}, u_{0}, \tau_{0}, p_{0}, \bar{p}\right)=\left(\tau_{0}, p_{0}, u_{0}, \tau_{0}, p_{0}, u_{0}-\left(a_{0}+b_{0}\right)\right)=\left(U_{l}^{0}, U_{r}^{0}\right) .
$$

Hence there exist neighborhoods $\mathfrak{N}$ of $\left(\tau_{0}, p_{0}, u_{0}, \tau_{0}, p_{0}, \tilde{p}\right)$ and $\Re$ of $\left(U_{l}^{0}\right.$, $\left.U_{r}^{0}\right)$ such that

$$
\left(\tau_{l}, p_{l}, u_{l}, \tau_{r}, p_{r}, p\right) \in \Re \Rightarrow\left(\tau_{l}, p_{l}, \tau_{r}, p_{r}, p\right) \in \mathscr{W},
$$

and $\left.\Psi\right|_{\Re}$ is a $C^{1}$ diffeomorphism of $\Re$ onto $\Re$. Thus, for any data $\left(U_{l}\right.$, $\left.U_{r}\right) \in \Re$, there exists a number $p$ such that

$$
\begin{gathered}
\left(\tau_{l}, p_{l}, \tau_{r}, p_{r}, p\right) \in \mathscr{W}, \\
u_{r}=u_{l}-\left(a_{r}+b_{l}\right)
\end{gathered}
$$

(the latter by (5.33)); $p$ depends continuously on $\left(U_{l}, U_{r}\right)$, and $p=\bar{p}$ when $\left(U_{l}, U_{r}\right)=\left(U_{l}^{0}, U_{r}^{0}\right)$. Moreover, by (5.34) there is a number $q$, also depending continuously on $\left(U_{l}, U_{r}\right) \in \mathcal{R}$, such that (5.30) holds; $q=\bar{p}$ when $\left(U_{l}\right.$, $\left.U_{r}\right)=\left(U_{l}^{0}, U_{r}^{9}\right)$. Therefore whenever $\left(U_{l}, U_{r}\right) \in \mathcal{X}$, numbers $p$ and $q$ exist such that the R. P. with data $\left(U_{l}, U_{r}\right)$ has the two distinct solutions (5.27) and (5.29); as $\left(U_{l}, U_{r}\right) \rightarrow\left(U_{l}^{0}, U_{r}^{0}\right)$, the constant states of the solutions (5.27) and (5.29) approach the respective constant states of the solutions (5.21), since $p$, $q \rightarrow \bar{p}$.

Lemmas 5.2, 5.3 and 5.5 give sufficient conditions for the occurrence of type I and type II nonuniqueness and their stability. Thus for our final result-that when WEAK fails we can get type I and type II nonuniqueness simultaneously, as well as their stability, for the same initial data-we must 
construct data $\left(U_{l}^{0}, U_{r}^{0}\right)$ that at once satisfy the hypotheses of Lemmas 5.2-5.3 and those of Lemma 5.5.

THEOREM 5.6. Suppose e violates WEAK. Then there exist data $\left(U_{l}^{0}, U_{r}^{0}\right)$ for which both 3-fold type I nonuniqueness and type II nonuniqueness hold, and the 5-fold nonuniqueness is stable.

The proof, which uses Lemma 4.14 , is long but rather routine, and hence is omitted.

\section{Examples.}

6.1. Energy functions that distinguish the conditions WEAK, MEDIUM and STRONG. All energy functions $e$ considered in this subsection are assumed to satisfy

$$
\lim _{\tau \rightarrow+\infty} e(\tau, S)=0 .
$$

We shall focus throughout this section on the one-parameter family of functions $g(\cdot, S),-\infty<S<+\infty$; each function is defined on $(0,+\infty)$ with $\tau$ as independent variable. To see what properties these functions have in general, let us fix $S \in(-\infty,+\infty)$ and define $\phi(\tau)=g(\tau, S)(\tau>0)$. We see immediately from (1.3), (1.5) and (1.6) that

$$
\phi>0, \quad \phi^{\prime}<0, \quad \phi^{\prime \prime}>0 \text {. }
$$

In addition, the following are easily verified:

$$
\begin{aligned}
\int_{1}^{+\infty} \phi & <+\infty \\
e(\tau, S) & =\int_{\tau}^{+\infty} g(v, S) d v \\
\lim _{\tau \rightarrow 0^{+}} \phi(\tau) & =+\infty
\end{aligned}
$$

Note also that $\phi$ is $C^{2}$.

Motivated by the fact that the functions $g(\cdot, S)$ always satisfy (6.2), (6.3) and (6.5), we make

Definition 6.1. Let $\mathscr{P}$ be the set of all $C^{2}$ functions $f$ defined on $(0,+\infty)$ for which $f>0, f^{\prime}<0, f^{\prime \prime}>0, \int_{1}^{+\infty} f<+\infty$ and $\lim _{\tau \rightarrow 0^{+}} f(\tau)=+\infty$.

“ $\mathcal{P}$ " signifies pressure. We have just proved

$$
g(\cdot, S) \in \mathcal{P} \quad(-\infty<S<+\infty),
$$

for every energy function $e$. (Remember that $e$ always satisfies (6.1) in this subsection.) In what follows, we shall construct and analyze one-parameter families $g(\cdot, S),-\infty<S<+\infty$, and get our energy functions via (6.4).

LeMma 6.2. Suppose e satisfies (1.2)-(1.9) and (6.1). Suppose also that $g(\cdot$, $S) \in \mathscr{P}$ for all $S$. Then e satisfies (1.10); hence $e$ is an energy function. 
Proof. Fix $p>0$. We first prove

$$
\lim _{\tau \rightarrow 0^{+}} S(\tau, p)=-\infty \text {. }
$$

(See (1.7)-(1.11) ff.) Let $S_{0} \in(-\infty,+\infty)$. Since $g\left(\cdot, S_{0}\right) \in \mathcal{P}$, we may choose $\tau_{0}>0$ such that $g\left(\tau_{0}, S_{0}\right)=p$. Let $\tau \in\left(0, \tau_{0}\right)$ and suppose $S(\tau$, $p) \geqslant S_{0}$. Then $p=g(\tau, S(\tau, p))>g\left(\tau_{0}, S(\tau, p)\right) \geqslant g\left(\tau_{0}, S_{0}\right)=p$, a contradiction. Hence $S(\tau, p)<S_{0}$. Thus (6.7) is proved.

Now let $\varepsilon>0$. Choose $\tau_{1}>0$ so that $\tau_{1} p \leqslant \frac{1}{2} \varepsilon$. Fix any $S_{1}$ and note that since $g\left(\cdot, S_{1}\right) \in \mathcal{P}$,

$$
\int_{\tau_{1}}^{+\infty} g\left(\cdot, S_{1}\right)<+\infty
$$

By (1.7), $g(\cdot, S)<g\left(\cdot, S_{1}\right)$ whenever $S<S_{1}$. By $(1.8), g(\cdot, S) \rightarrow 0$ pointwise on $\left[\tau_{1},+\infty\right)$ as $S \rightarrow-\infty$. Hence by the Lebesgue convergence theorem,

$$
\lim _{S \rightarrow-\infty} \int_{\tau_{1}}^{+\infty} g(\cdot, S)=0 .
$$

Thus $\exists M>0$ such that

$$
\int_{\tau_{1}}^{+\infty} g(\cdot, S)<\frac{1}{2} \varepsilon \quad \text { whenever } S<-M .
$$

By (6.7), $\exists \delta \in\left(0, \tau_{1}\right)$ such that $S(\tau, p)<-M$ whenever $\tau \in(0, \delta)$. Let $\tau \in(0, \delta)$. By $(1.5), g(v, S(\tau, p)) \leqslant g(\tau, S(\tau, p))=p$ whenever $v \geqslant \tau$. Then from (6.1) and (6.8) we get

$$
\begin{aligned}
e(\tau, p) & =\int_{\tau}^{+\infty} g(v, S(\tau, p)) d v \\
& =\int_{\tau}^{\tau_{1}} g(v, S(\tau, p)) d v+\int_{\tau_{1}}^{+\infty} g(v, S(\tau, p)) d v \\
& <\int_{\tau}^{\tau_{1}} p d v+\frac{1}{2} \varepsilon \\
& =\left(\tau_{1}-\tau\right) p+\frac{1}{2} \varepsilon<\varepsilon .
\end{aligned}
$$

Thus (1.10) is proved.

Proposition 6.3. Let $\phi \in \mathcal{P}$. Let $\theta$ be a $C^{3}$ function mapping $(-\infty,+\infty)$ onto $(0,+\infty)$ such that $\theta^{\prime}>0$. Define

$$
e(\tau, S)=\theta(S) \int_{\tau}^{+\infty} \phi \quad(\tau>0,-\infty<S<+\infty) .
$$

Then $e$ is an energy function satisfying MEDIUM. $\phi$ can be chosen so that $e$ violates STRONG.

Proof. Clearly $e$ is $C^{3}$ in $(\tau, S)$ and $e>0$. Define $g=-e_{\tau}$; then, using Lemma 6.2 , it is easy to verify that $e$ is an energy function. 
Let $\tau, p>0$. Let $S$ satisfy $g(\tau, S)=p$; then

$$
\theta(S) \phi(\tau)=p .
$$

Moreover, $e(\tau, p)=e(\tau, S)=\theta(S) \int_{\tau}^{+\infty} \phi$; this, together with (6.9), gives

$$
e(\tau, p)=\frac{p}{\phi(\tau)} \int_{\tau}^{+\infty} \phi \quad(\tau, p>0)
$$

We also have

$$
\begin{aligned}
g_{\tau}(\tau, p) & =g_{\tau}(\tau, S)=\theta(S) \phi^{\prime}(\tau), \\
\frac{\partial}{\partial p} e(\tau, p) & =\frac{1}{\phi(\tau)} \int_{\tau}^{+\infty} \phi .
\end{aligned}
$$

Using Proposition 1.5, (6.9)-(6.12) and Lemma 1.6, we see that $e$ satisfies MEDIUM.

Finally,

$$
\begin{aligned}
\text { STRONG } & \Leftrightarrow \frac{\partial}{\partial p} e(\tau, p) \geqslant \frac{p}{-g_{\tau}} \Leftrightarrow \frac{1}{\phi(\tau)} \int_{\tau}^{+\infty} \phi \geqslant \frac{\theta(S) \phi(\tau)}{-\theta(S) \phi^{\prime}(\tau)} \\
& \Leftrightarrow \int_{\tau}^{+\infty} \phi \geqslant \phi(\tau)\left(\frac{\phi(\tau)}{-\phi^{\prime}(\tau)}\right) .
\end{aligned}
$$

Referring to Figure 14, we see that $\phi(\tau)\left(\phi(\tau) /-\phi^{\prime}(\tau)\right)$ is the area of the rectangle $A B C D$, whereas $\phi$ can be chosen so as to make $\int_{\tau}^{+\infty} \phi$ arbitrarily close to the area of $\triangle A B D$. In particular

$$
\int_{\tau}^{+\infty} \phi<\phi(\tau)\left(\frac{\phi(\tau)}{-\phi^{\prime}(\tau)}\right)
$$

for suitable choice of $\phi$; STRONG fails for such $\phi$.

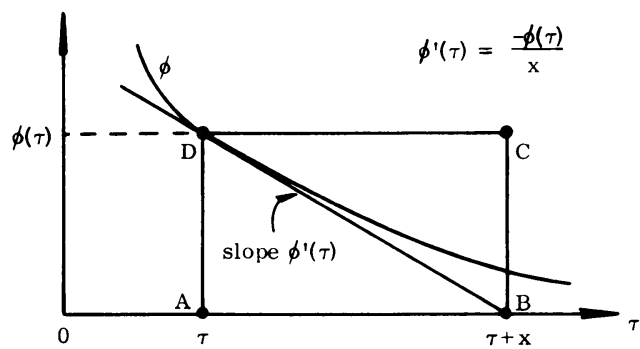

Figure 14. $\phi$ can be chosen so that STRONG fails.

As a corollary we have

THEOREM 6.4. There exists an energy function that satisfies MEDIUM but violates STRONG. 
To get an example of an energy function that satisfies WEAK but violates MEDIUM, we shall begin by establishing a criterion for WEAK in terms of the one-parameter family $g(\cdot, S),-\infty<S<+\infty$, of elements of $\mathscr{P}$.

Definition 6.5. Let $f, g \in \mathscr{P}$. We say $f \underset{\sim}{\sim} g$ iff $f \leqslant g$ and for any $\tau_{1}, \tau_{2}$, $p>0$ such that $\tau_{1} \leqslant \tau_{2}$ and $f\left(\tau_{1}\right)=g\left(\tau_{2}\right)=p$, we have

$$
\int_{\tau_{2}}^{+\infty} g \geqslant \int_{\tau_{1}}^{+\infty} f-\left(\tau_{2}-\tau_{1}\right) \frac{1}{2} p \text {. }
$$

Proposition 6.6. e satisfies WEAK iff for all $S_{1}, S_{2} \in(-\infty,+\infty), S_{1}<S_{2}$ implies $g\left(\cdot, S_{1}\right) \stackrel{W}{\sim} g\left(\cdot, S_{2}\right)$.

PROof. Straightforward.

PROPOSITION 6.7. The relation $\stackrel{W}{\sim}$ has the following properties.

(a) $f \stackrel{W}{\sim}$ g and $\alpha>0 \Rightarrow \alpha f \stackrel{W}{\sim} \alpha g$.

(b) $f \in \mathscr{P}$ and $0<\alpha \leqslant \beta \Rightarrow \alpha f \stackrel{W}{\sim} \beta$.

(c) $f \stackrel{W}{\sim}$ and $g \stackrel{W}{\sim} h \Rightarrow f \stackrel{W}{\sim}$.

Proof. (a) and (c) are straightforward; for (b), use Propositions 6.3 and 6.6.

LEMma 6.8. Let $f, g \in \mathscr{P}$ with $f<g$, and define $\pi(\cdot, \lambda)=(1-\lambda) f+\lambda g$. Suppose for all $\tau>0$,

$$
\frac{2}{g(\tau)-f(\tau)} \int_{\tau}^{+\infty}(g-f) \geqslant\left\{\begin{array}{c}
\frac{f(\tau)}{-f^{\prime}(\tau)}, \\
\frac{g(\tau)}{-g^{\prime}(\tau)} .
\end{array}\right.
$$

Then $\pi\left(\cdot, \lambda_{1}\right) \stackrel{W}{\sim} \pi\left(\cdot, \lambda_{2}\right)$ whenever $0 \leqslant \lambda_{1} \leqslant \lambda_{2} \leqslant 1$.

Proof. Since $f, g \in \mathcal{P}$, we may define

$$
\varepsilon(\tau, \lambda)=\int_{\tau}^{+\infty} \pi(v, \lambda) d v=(1-\lambda) \int_{\tau}^{+\infty} f+\lambda \int_{\tau}^{+\infty} g .
$$

Then $\varepsilon$ is $C^{3}$ in $(\tau, \lambda)$. We have

$$
\pi_{\lambda}(\tau, \lambda)=g(\tau)-f(\tau)>0
$$

so we can define for any $\tau, p>0, \varepsilon(\tau, p)=\varepsilon(\tau, \lambda)$ where $\lambda$ satisfies $\pi(\tau$, $\lambda)=p$. (We then denote $\lambda=\lambda(\tau, p)$.) Similarly, define $\pi_{\tau}(\tau, p)=\pi_{\tau}(\tau, \lambda(\tau$, $p))(\tau, p>0)$. Now let $\tau, p>0$, and let $\lambda=(p-f(\tau)) /(g(\tau)-f(\tau))$. Then

$$
1-\lambda=\frac{g(\tau)-p}{g(\tau)-f(\tau)}
$$


and

$$
\pi(\tau, \lambda)=(1-\lambda) f(\tau)+\lambda g(\tau)=p,
$$

whence

$$
\lambda(\tau, p)=\frac{p-f(\tau)}{g(\tau)-f(\tau)} \quad(\tau, p>0)
$$

Also note that

$$
\pi_{\tau}(\tau, \lambda)=(1-\lambda) f^{\prime}(\tau)+\lambda g^{\prime}(\tau)
$$

We assert that

$$
\frac{\partial}{\partial p} \varepsilon(\tau, p) \geqslant \frac{-p}{2 \pi_{\tau}(\tau, p)} \quad(f(\tau) \leqslant p \leqslant g(\tau)) .
$$

For by (6.13) and (6.14),

$$
\begin{aligned}
\varepsilon(\tau, p) & =\varepsilon(\tau, \lambda(\tau, p)) \\
& =\frac{g(\tau)-p}{g(\tau)-f(\tau)} \int_{\tau}^{+\infty} f+\frac{p-f(\tau)}{g(\tau)-f(\tau)} \int_{\tau}^{+\infty} g,
\end{aligned}
$$

whence

$$
\frac{\partial}{\partial p} \varepsilon(\tau, p)=\frac{1}{g(\tau)-f(\tau)} \int_{\tau}^{+\infty}(g-f) .
$$

Let us temporarily hold $\tau$ constant; define

$$
\Phi(p)=\frac{-p}{(g(\tau)-p) f^{\prime}(\tau)+(p-f(\tau)) g^{\prime}(\tau)} .
$$

$\Phi$ is defined on an open interval $I$ containing $[f(\tau), g(\tau)]$. For $p \in I,(6.14)$ and (6.15) yield

$$
\begin{aligned}
& -\frac{p}{2 \pi_{\tau}(\tau, p)} \\
& =\frac{-p}{2\left[(g(\tau)-p) f^{\prime}(\tau) /(g(\tau)-f(\tau))+(p-f(\tau)) g^{\prime}(\tau) /(g(\tau)-f(\tau))\right]} \\
& =\frac{1}{2}(g(\tau)-f(\tau)) \Phi(p) .
\end{aligned}
$$

We have

$$
\Phi^{\prime}(p)=\frac{f(\tau) g^{\prime}(\tau)-g(\tau) f^{\prime}(\tau)}{\left[(g(\tau)-p) f^{\prime}(\tau)+(p-f(\tau)) g^{\prime}(\tau)\right]^{2}} \quad(p \in I) ;
$$

thus, depending on the sign of $f(\tau) g^{\prime}(\tau)-g(\tau) f^{\prime}(\tau)$, either $\Phi^{\prime} \geqslant 0$ on $I$ or $\Phi^{\prime} \leqslant 0$ on $I$. Hence $\Phi$ attains its maximum on $[f(\tau), g(\tau)]$ at an endpoint, $f(\tau)$ 
or $g(\tau)$. But

$$
\Phi(f(\tau))=\frac{-f(\tau)}{(g(\tau)-f(\tau)) f^{\prime}(\tau)}, \quad \Phi(g(\tau))=\frac{-g(\tau)}{(g(\tau)-f(\tau)) g^{\prime}(\tau)} .
$$

Using (6.18), we conclude that $-p / 2 \pi_{\tau}(\tau, p)$ attains its maximum value for $p \in[f(\tau), g(\tau)]$ either at $p=f(\tau)$ or $p=g(\tau)$, and its maximum value is either $g(\tau) /-2 g^{\prime}(\tau)$ or $f(\tau) /-2 f^{\prime}(\tau)$. But by (6.17) and the hypothesis, $\partial \varepsilon(\tau$, $p) / \partial p$ is always greater than or equal to each of these values; hence (6.16) holds.

Now proceed by analogy with the proofs of Proposition 1.5 and Proposition 6.6.

THEOREM 6.9. There exists an energy function e that satisfies WEAK but violates MEDIUM.

Proof. Choose any $\phi_{0} \in \mathcal{P} \cap C^{3}(0,+\infty)$ that satisfies $\phi_{0}^{\prime \prime \prime}<0$. Define

$$
\phi(\tau, S)=\phi_{0}(\tau)\left(1-S \phi_{0}^{\prime}(\tau)\right) \quad(\tau>0,-\infty<S<+\infty) .
$$

Then

$$
|\phi(\tau, S)| \leqslant \phi_{0}(\tau)+|S| \phi_{0}(\tau)\left(-\phi_{0}^{\prime}(\tau)\right) \quad(\tau>0) .
$$

Now

$$
\begin{array}{r}
\int_{\tau}^{M}-\phi_{0}(v) \phi_{0}^{\prime}(v) d v=-\left.\frac{1}{2} \phi_{0}(v)^{2}\right|_{v=\tau} ^{v=M}=\frac{1}{2}\left[\phi_{0}(\tau)^{2}-\phi_{0}(M)^{2}\right] \\
(\tau, M>0) .
\end{array}
$$

With $\tau=1$, (6.21) yields

$$
\int_{1}^{+\infty}-\phi_{0}(v) \phi_{0}^{\prime}(v) d v<+\infty
$$

Since also $\int_{1}^{+\infty} \phi_{0}<+\infty$, we obtain from (6.20)

$$
\int_{1}^{+\infty}|\phi(\cdot, S)|<+\infty
$$

Thus we may define

$$
\varepsilon(\tau, S)=\int_{\tau}^{+\infty} \phi(v, S) d v \quad(\tau>0)
$$

in fact, by (6.19) and (6.21) we have

$$
\varepsilon(\tau, S)=\int_{\tau}^{+\infty} \phi_{0}+\frac{S}{2} \phi_{0}(\tau)^{2} \quad(\tau>0),
$$

which shows $\varepsilon$ is $C^{3}$ in $(\tau, S)$. By $(6.23), \varepsilon_{\tau}(\tau, S)=-\phi(\tau, S)(\tau>0)$. By (6.19), $\phi_{S}(\tau, S)=-\phi_{0}(\tau) \phi_{0}^{\prime}(\tau)>0(\tau>0)$; thus we may, as usual, define

$$
\varepsilon(\tau, p)=\varepsilon(\tau, S) \quad(\tau>0,-\infty<p<+\infty),
$$

where $S=\hat{S}(\tau, p)$ is the unique solution of $\phi(\tau, S)=p$. 
We assert that

$$
\phi(\cdot, S) \in \mathscr{\rho} \quad(S>0) .
$$

Clearly $\phi(\tau, S)>0(\tau>0, S \geqslant 0)$. Differentiating (6.19) with respect to $\tau$ gives

$$
\phi_{\tau}(\tau, S)=\phi_{0}^{\prime}(\tau)-S\left[\phi_{0}(\tau) \phi_{0}^{\prime \prime}(\tau)+\phi_{0}^{\prime}(\tau)^{2}\right] \quad(\tau>0),
$$

whence $\phi_{\tau}(\cdot, S)<0(S \geqslant 0)$. Differentiating (6.26), we get $\phi_{\tau \tau}(\tau, S)=\phi_{0}^{\prime \prime}(\tau)-S\left[\phi_{0}(\tau) \phi_{0}^{\prime \prime \prime}(\tau)+3 \phi_{0}^{\prime}(\tau) \phi_{0}^{\prime \prime}(\tau)\right]>0 \quad(\tau>0, S \geqslant 0)$.

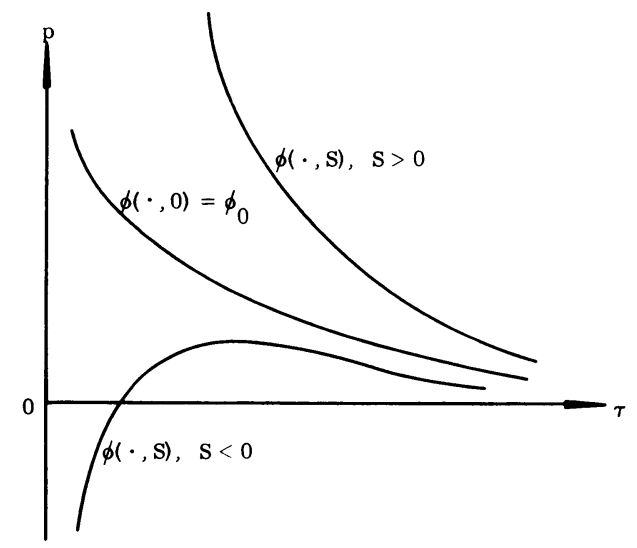

FIGURE 15 . The family $\phi(\cdot, S),-\infty<S<+\infty$.

By (6.22), $\int_{1}^{+\infty} \phi(\cdot, S)<+\infty \quad(S \geqslant 0)$. Since $\phi(\cdot, S) \geqslant \phi_{0} \quad(S \geqslant 0)$, $\lim _{\tau \rightarrow 0^{+}} \phi(\tau, S)=+\infty(S \geqslant 0)$. Thus $(6.25)$ is proved.

Now for $\tau>0$ and all $S$, we have

$$
\int_{\tau}^{+\infty} \phi(\cdot, S)-\int_{\tau}^{+\infty} \phi_{0}=\frac{S}{2} \phi_{0}(\tau)^{2}
$$

by (6.23) and (6.24); and by (6.19),

$$
\phi(\tau, S)-\phi_{0}(\tau)=-S \phi_{0}(\tau) \phi_{0}^{\prime}(\tau)
$$

Thus

$$
\frac{\int_{\tau}^{+\infty} \phi(\cdot, S)-\int_{\tau}^{+\infty} \phi_{0}}{\phi(\tau, S)-\phi_{0}(\tau)}=-\frac{1}{2} \frac{\phi_{0}(\tau)}{\phi_{0}^{\prime}(\tau)} \quad(\tau>0, S \neq 0) .
$$

For $S=1,(6.27)$ becomes

$$
\frac{2}{\phi(\tau, 1)-\phi_{0}(\tau)} \int_{\tau}^{+\infty}\left(\phi(\cdot, 1)-\phi_{0}\right)=\frac{\phi_{0}(\tau)}{-\phi_{0}^{\prime}(\tau)} \quad(\tau>0) .
$$


Now observe that for $\tau, S>0$,

$$
\begin{aligned}
\frac{\phi_{0}(\tau)}{-\phi_{0}^{\prime}(\tau)} & \geqslant \frac{\phi(\tau, S)}{-\phi_{\tau}(\tau, S)} \Leftrightarrow-\phi_{0}(\tau) \phi_{\tau}(\tau, S) \geqslant-\phi(\tau, S) \phi_{0}^{\prime}(\tau) \\
& \Leftrightarrow-\phi_{0}(\tau)\left[\phi_{0}^{\prime}(\tau)-S\left(\phi_{0}(\tau) \phi_{0}^{\prime \prime}(\tau)+\phi_{0}^{\prime}(\tau)^{2}\right)\right] \\
& \geqslant-\phi_{0}(\tau)\left(1-S \phi_{0}^{\prime}(\tau)\right) \phi_{0}^{\prime}(\tau) \\
& \Leftrightarrow S \phi_{0}(\tau)\left(\phi_{0}(\tau) \phi_{0}^{\prime \prime}(\tau)+\phi_{0}^{\prime}(\tau)^{2}\right) \geqslant S \phi_{0}(\tau) \phi_{0}^{\prime}(\tau)^{2} \Leftrightarrow \phi_{0}(\tau) \phi_{0}^{\prime \prime}(\tau) \geqslant 0,
\end{aligned}
$$

by (6.19) and (6.26). Hence

$$
\frac{\phi_{0}(\tau)}{-\phi_{0}^{\prime}(\tau)} \geqslant \frac{\phi(\tau, S)}{-\phi_{\tau}(\tau, S)} \quad(\tau, S>0) .
$$

It follows from (6.28) and (6.29) that $\forall \tau>0$,

$$
\frac{2}{\phi(\tau, 1)-\phi_{0}(\tau)} \int_{\tau}^{+\infty}\left(\phi(\cdot, 1)-\phi_{0}\right) \geqslant\left\{\begin{array}{c}
\frac{\phi_{0}(\tau)}{-\phi_{0}^{\prime}(\tau)}, \\
\frac{\phi(\tau, 1)}{-\phi_{\tau}(\tau, 1)}
\end{array}\right.
$$

Note that $\phi(\cdot, 1)>\phi_{0}$, so Lemma 6.8 applies. Define

$$
\pi(\tau, \lambda)=(1-\lambda) \phi_{0}(\tau)+\lambda \phi(\tau, 1) \quad(\tau>0,-\infty<\lambda<+\infty)
$$

then by Lemma 6.8 ,

$$
\pi\left(\cdot, \lambda_{1}\right) \stackrel{W}{\sim} \pi\left(\cdot, \lambda_{2}\right) \quad\left(0 \leqslant \lambda_{1} \leqslant \lambda_{2}<1\right) .
$$

But

$$
\begin{aligned}
\pi(\tau, \lambda) & =(1-\lambda) \phi_{0}(\tau)+\lambda \phi_{0}(\tau)\left(1-\phi_{0}^{\prime}(\tau)\right) \\
& =\phi_{0}(\tau)\left(1-\lambda+\lambda\left(1-\phi_{0}^{\prime}(\tau)\right)\right)=\phi(\tau, \lambda)
\end{aligned}
$$

hence

$$
\phi\left(\cdot, S_{1}\right) \stackrel{W}{\sim} \phi\left(\cdot, S_{2}\right) \quad\left(0 \leqslant S_{1} \leqslant S_{2} \leqslant 1\right) .
$$

Now let $\tau>0$; we shall compute $\partial \varepsilon(\tau, p) /\left.\partial p\right|_{\left(\tau, \phi_{0}(\tau)\right)}$. Since $\phi(\tau, 0)=\phi_{0}(\tau)$, $\hat{S}\left(\tau, \phi_{0}(\tau)\right)=0$, so

$$
\varepsilon\left(\tau, \phi_{0}(\tau)\right)=\varepsilon(\tau, 0)=\int_{\tau}^{+\infty} \phi(v, 0) d v=\int_{\tau}^{+\infty} \phi_{0} .
$$

Also, with $p=\phi(\tau, S)$, i.e., $S=\hat{S}(\tau, p)$ ( $\tau$ fixed), $S \rightarrow 0$ as $p \rightarrow \phi_{0}(\tau)$ and 
vice-versa. Thus

$$
\begin{aligned}
\left.\frac{\partial}{\partial p} \varepsilon(\tau, p)\right|_{\left(\tau, \phi_{0}(\tau)\right)} & =\lim _{p \rightarrow \phi_{0}(\tau)} \frac{\varepsilon(\tau, p)-\varepsilon\left(\tau, \phi_{0}(\tau)\right)}{p-\phi_{0}(\tau)} \\
& =\lim _{p \rightarrow \phi_{0}(\tau)} \frac{\varepsilon(\tau, \hat{S}(\tau, p))-\varepsilon\left(\tau, \phi_{0}(\tau)\right)}{\phi(\tau, \hat{S}(\tau, p))-\phi_{0}(\tau)} \\
& =\lim _{S \rightarrow 0} \frac{\varepsilon(\tau, S)-\int_{\tau}^{+\infty} \phi_{0}}{\phi(\tau, S)-\phi_{0}(\tau)}=-\frac{1}{2} \frac{\phi_{0}(\tau)}{\phi_{0}^{\prime}(\tau)},
\end{aligned}
$$

by (6.27). We define $\phi_{\tau}(\tau, p)=\phi_{\tau}(\tau, \hat{S}(\tau, p))$; then, with $\phi_{0}(\tau)$ understood to be $p$, we have

$$
\phi_{\tau}\left(\tau, \phi_{0}(\tau)\right)=\phi_{\tau}(\tau, 0)=\phi_{0}^{\prime}(\tau)
$$

By (6.23), (6.25) and Lemma 1.6,

$$
\phi(\tau, S)^{2}<-2 \phi_{\tau}(\tau, S) \varepsilon(\tau, S) \quad(S \geqslant 0)
$$

by (6.32), this becomes for $S=0$,

$$
\phi_{0}(\tau)^{2}<-2 \phi_{\tau}\left(\tau, \phi_{0}(\tau)\right) \varepsilon\left(\tau, \phi_{0}(\tau)\right) .
$$

Using (6.32), (6.31) may be written

$$
\begin{aligned}
\left.\frac{\partial}{\partial p} \varepsilon(\tau, p)\right|_{\left(\tau, \phi_{0}(\tau)\right)} & \\
= & \frac{2 \varepsilon\left(\tau, \phi_{0}(\tau)\right) \phi_{0}(\tau)}{-\phi_{\tau}\left(\tau, \phi_{0}(\tau)\right) \cdot 2 \varepsilon\left(\tau, \phi_{0}(\tau)\right)-\phi_{\tau}\left(\tau, \phi_{0}(\tau)\right) \cdot 2 \varepsilon\left(\tau, \phi_{0}(\tau)\right)} ;
\end{aligned}
$$

then using (6.33) and dividing numerator and denominator by $\phi_{0}(\tau)$, we get

$$
\begin{array}{r}
\left.\frac{\partial}{\partial p} \varepsilon(\tau, p)\right|_{\left(\tau, \phi_{0}(\tau)\right)}<\frac{2 \varepsilon\left(\tau, \phi_{0}(\tau)\right)}{-\phi_{\tau}\left(\tau, \phi_{0}(\tau)\right) \cdot\left(2 \varepsilon\left(\tau, \phi_{0}(\tau)\right) / \phi_{0}(\tau)\right)+\phi_{0}(\tau)} \\
(\tau>0) .
\end{array}
$$

Now fix $\tau_{1}>0$. Since $\varepsilon(\tau, p), \phi_{\tau}(\tau, p)$ and $\partial \varepsilon(\tau, p) / \partial p$ are continuous in $(\tau$, $p$ ), it follows from (6.34) with $\tau=\tau_{1}$ that

$$
\left.\frac{\partial}{\partial p} \varepsilon(\tau, p)\right|_{\left(\tau_{1}, p_{1}\right)}<\frac{2 \varepsilon\left(\tau_{1}, p_{1}\right)}{-\phi_{\tau}\left(\tau_{1}, p_{1}\right) \cdot\left(2 \varepsilon\left(\tau_{1}, p_{1}\right) / p_{1}\right)+p_{1}}
$$

for some $p_{1}$ with $\phi_{0}\left(\tau_{1}\right)<p_{1}<\phi\left(\tau_{1}, 1\right)$. Let $S_{1}$ satisfy $\phi\left(\tau_{1}, S_{1}\right)=p_{1}$; then $0<S_{1}<1$. Choose $\delta>0$ such that $\delta<S_{1}<1-\delta$. Let $\lambda$ be a $C^{3}$ function defined on $(-\infty,+\infty)$ such that

$$
\begin{array}{ll}
\lambda^{\prime}(S)>0 & (-\delta<S<1+\delta), \\
\lambda(S)=0 & (S \leqslant-\delta),
\end{array}
$$




$$
\begin{array}{ll}
\lambda(S)=1 & (S \geqslant 1+\delta), \\
\lambda(S)=S & (\delta \leqslant S \leqslant 1-\delta) .
\end{array}
$$

Let $\alpha$ be a $C^{3}$ function defined on $(-\infty,+\infty)$ such that $\alpha>0$ and

$$
\begin{array}{ll}
\alpha^{\prime}(S)>0 & (S<0, S>1), \\
\alpha(S)=1 \quad(0 \leqslant S \leqslant 1), \\
\lim _{S \rightarrow-\infty} \alpha(S)=0, \\
\lim _{S \rightarrow+\infty} \alpha(S)=+\infty .
\end{array}
$$

Define

$$
\begin{aligned}
& g(\tau, S)=\alpha(S) \phi(\tau, \lambda(S)) \quad(\tau>0,-\infty<S<+\infty), \\
& e(\tau, S)=\alpha(S) \varepsilon(\tau, \lambda(S)) \quad(\tau>0,-\infty<S<+\infty) .
\end{aligned}
$$

(See (6.19) and (6.23).) Then it is easily verified, using Lemma 6.2, that $e$ is an energy function.

To establish WEAK, we shall apply Proposition 6.6. Let $S_{1}, S_{2} \in(-\infty$, $+\infty)$ with $S_{1}<S_{2}$. Then certainly $0 \leqslant \lambda\left(S_{1}\right) \leqslant \lambda\left(S_{2}\right) \leqslant 1$, so by $(6.30)$,

$$
\phi\left(\cdot, \lambda\left(S_{1}\right)\right) \stackrel{\boldsymbol{w}}{\sim} \phi\left(\cdot, \lambda\left(S_{2}\right)\right)
$$

Moreover, $0<\alpha\left(S_{1}\right) \leqslant \alpha\left(S_{2}\right)$, so by Proposition 6.7(a),

$$
\alpha\left(S_{1}\right) \phi\left(\cdot, \lambda\left(S_{1}\right)\right) \stackrel{W}{\sim} \alpha\left(S_{1}\right) \phi\left(\cdot, \lambda\left(S_{2}\right)\right) ;
$$

and by Proposition 6.7(b),

$$
\alpha\left(S_{1}\right) \phi\left(\cdot, \lambda\left(S_{2}\right)\right) \stackrel{W}{\sim} \alpha\left(S_{2}\right) \phi\left(\cdot, \lambda\left(S_{2}\right)\right) .
$$

Applying Proposition 6.7(c) to (6.46) and (6.47), we obtain

$$
\alpha\left(S_{1}\right) \phi\left(\cdot, \lambda\left(S_{1}\right)\right) \stackrel{W}{\sim} \alpha\left(S_{2}\right) \phi\left(\cdot, \lambda\left(S_{2}\right)\right),
$$

i.e.,

$$
g\left(\cdot, S_{1}\right) \stackrel{\boldsymbol{W}}{\sim} g\left(\cdot, S_{2}\right)
$$

Since $S_{1}$ and $S_{2}$ were arbitrary, $e$ satisfies WEAK by Proposition 6.6.

By (6.39) and (6.41), $\lambda(S)=S$ and $\alpha(S)=1$ whenever $\delta<S<1-\delta$; hence

$$
\begin{aligned}
& g(\tau, S)=\phi(\tau, S), \\
& e(\tau, S)=\varepsilon(\tau, S),
\end{aligned}
$$

whenever $(\tau, S) \in(0,+\infty) \times(\delta, 1-\delta)$. Since $\left(\tau_{1}, S_{1}\right) \in(0,+\infty) \times(\delta$, $1-\delta)$, we see that $g=\phi$ and $e=\varepsilon$ on a neighborhood $N$ of $\left(\tau_{1}, S_{1}\right)$. Define $\Phi(\tau, S)=(\tau, g(\tau, S)), \Psi(\tau, S)=(\tau, \phi(\tau, S))$; then

$$
\Phi=\Psi \text { on } N,
$$


and the Jacobian determinant $J_{\Psi}\left(\tau_{1}, S_{1}\right)=\phi_{S}\left(\tau_{1}, S_{1}\right)>0$. Hence by making $N$ smaller we may assume $\Phi$ maps $N$ (injectively) onto an open set $M$ containing $\left(\tau_{1}, p_{1}\right)$. (Recall that $\phi\left(\tau_{1}, S_{1}\right)=p_{1}$.) For $(\tau, p) \in M$, we have

$$
\Phi^{-1}(\tau, p)=(\tau, S(\tau, p)), \quad \Psi^{-1}(\tau, p)=(\tau, \hat{S}(\tau, p)) ;
$$

hence by (6.50), $S=\hat{S}$ on $M$. Then by (6.49), $\forall(\tau, p) \in M, e(\tau, p)=e(\tau$, $S(\tau, p))=\varepsilon(\tau, S(\tau, p))=\varepsilon(\tau, \hat{S}(\tau, p))=\varepsilon(\tau, p)$. From this it follows that

$$
\left.\frac{\partial}{\partial p} e(\tau, p)\right|_{\left(\tau_{1}, p_{1}\right)}=\left.\frac{\partial}{\partial p} \varepsilon(\tau, p)\right|_{\left(\tau_{1}, p_{1}\right)} .
$$

Also, by (6.48), $g_{\tau}\left(\tau_{1}, p_{1}\right)=g_{\tau}\left(\tau_{1}^{*}, S_{1}\right)=\phi_{\tau}\left(\tau_{1}, S_{1}\right)=\phi_{\tau}\left(\tau_{1}, p_{1}\right)$. From this, (6.51) and (6.35), we obtain

$$
\left.\frac{\partial}{\partial p} e(\tau, p)\right|_{\left(\tau_{1}, p_{1}\right)}<\frac{2 e\left(\tau_{1}, p_{1}\right)}{-g_{\tau}\left(\tau_{1}, p_{1}\right) \cdot\left(2 e\left(\tau_{1}, p_{1}\right) / p_{1}\right)+p_{1}} ;
$$

thus $e$ violates MEDIUM.

6.2. An energy function that violates WEAK.

THEOREM 6.10. There exists an energy function that violates WEAK.

Proof. For convenience, we shall set $v=1-\tau$. Fix $\alpha \in(0,1 / 2)$, and define

$$
f(v, S)=e^{v}+\alpha\left(1-e^{-S}\right) \exp \left[\alpha^{-1}(v+S)\right] .
$$

After some computation, we shall almost get the energy function we want by replacing " $v$ " by " $1-\tau$ ". We have

$$
\begin{aligned}
f_{v}(v, S) & =e^{v}+\left(1-e^{-S}\right) \exp \left[\alpha^{-1}(v+S)\right], \\
f_{v v}(v, S) & =e^{v}+\alpha^{-1}\left(1-e^{-S}\right) \exp \left[\alpha^{-1}(v+S)\right], \\
f_{v v v}(v, S) & =e^{v}+\alpha^{-2}\left(1-e^{-S}\right) \exp \left[\alpha^{-1}(v+S)\right]
\end{aligned}
$$

From (6.52) and (6.53) we see that $f, f_{v}, f_{v v}$ and $f_{v v v}$ have the form

$$
h(v, S)=e^{v}+\beta\left(1-e^{-S}\right) \exp \left[\alpha^{-1}(v+S)\right],
$$

where $0<\beta \leqslant \alpha^{-2}$. But $h(v, S)>0$ iff

$$
\exp \left[\left(1-\alpha^{-1}\right) v\right]>\beta\left(e^{-S}-1\right) e^{S / \alpha} \text {. }
$$

Also, since $1-1 / \alpha<0$,

$$
\exp \left[\left(1-\alpha^{-1}\right) v\right] \geqslant \exp \left(1-\alpha^{-1}\right) \quad \text { whenever } v \leqslant 1 .
$$

Choose $\delta_{1}>0$ such that

$$
\alpha^{-2}\left(e^{-S}-1\right) e^{S / \alpha}<\exp \left(1-\alpha^{-1}\right) \quad \text { whenever } S \geqslant-\delta_{1} .
$$


Combining (6.54)-(6.57), we conclude

$$
h(v, S)>0 \quad \text { whenever } v \leqslant 1, S \geqslant-\delta_{1},
$$

so that

$$
f, f_{v}, f_{v v}, f_{v v v}>0 \quad\left(v \leqslant 1, S \geqslant-\delta_{1}\right) .
$$

We also have

$$
\begin{aligned}
& f_{S}(v, S)=\exp \left[\alpha^{-1}(v+S)\right]\left[1-(1-\alpha) e^{-S}\right], \\
& f_{v S}(v, S)=\alpha^{-1} f_{S}(v, S) .
\end{aligned}
$$

Choose $\delta_{2}>0$ such that $(1-\alpha) e^{-S}<1$ whenever $S \geqslant-\delta_{2}$. Then

$$
f_{S}, f_{v S}>0 \quad\left(v \leqslant 1, S \geqslant-\delta_{2}\right) \text {. }
$$

Let $\delta$ be the smaller of $\delta_{1}$ and $\delta_{2}$. Then by (6.58) and (6.59),

$$
f, f_{v}, f_{v v}, f_{v v v}, f_{S}, f_{v S}>0 \quad(v \leqslant 1, S \geqslant-\delta) .
$$

Now define

$$
\varepsilon(\tau, S)=f(1-\tau, S) \quad(\tau \geqslant 0, S>-\delta) .
$$

Since $\tau \geqslant 0$ iff $1-\tau \leqslant 1$, we see that (6.60) yields

$\varepsilon>0, \quad \varepsilon_{\tau}<0, \quad \varepsilon_{\tau \tau}>0, \quad \varepsilon_{\tau \tau \tau}<0, \quad \varepsilon_{S}>0, \quad \varepsilon_{\tau S}<0 \quad(\tau \geqslant 0, S>-\delta)$,

which, except for the restriction on $S$, is equivalent to (1.2)-(1.7) for $\varepsilon$ in place of $e$. Let $\xi$ be a $C^{3}$ function defined on $(-\infty,+\infty)$ such that

$$
\begin{aligned}
\xi^{\prime} & >0, \\
\xi(S) & =S \quad(S>-\delta / 2), \\
\lim _{S \rightarrow-\infty} \xi(S) & =-\delta .
\end{aligned}
$$

Let $\theta$ be a $C^{3}$ function defined on $(-\infty,+\infty)$ such that $\theta>0$ and

$$
\begin{aligned}
\theta^{\prime} & \geqslant 0, \\
\theta(S) & =1 \quad(S>-\delta / 2), \\
\lim _{S \rightarrow-\infty} \theta(S) & =0 .
\end{aligned}
$$

Let $\gamma$ be a $C^{3}$ function defined on $(0,+\infty)$ such that

$$
\begin{aligned}
\gamma^{\prime} & \leqslant 0, \quad \gamma^{\prime \prime} \geqslant 0, \quad \gamma^{\prime \prime \prime} \leqslant 0, \\
\gamma(\tau) & =1 \quad(\tau>1-\delta / 2), \\
\lim _{\tau \rightarrow 0^{+}} \gamma(\tau) & =+\infty
\end{aligned}
$$

Define

$$
e(\tau, S)=\gamma(\tau) \theta(S) \varepsilon(\tau, \xi(S)) \quad(\tau>0,-\infty<S<+\infty) .
$$

Then $e$ is an energy function. (Use Lemma 6.2.) 
We shall prove that WEAK fails at $\tau=1, p=1$. For clarity, we let $\bar{p}=1$ throughout. Now let $\tau>1-\delta / 2$. Then $\tau-1>-\delta / 2$, and from (6.64), (6.67) and (6.70) we conclude $\xi(\tau-1)=\tau-1, \theta(\tau-1)=1, \gamma(\tau)=1$ and $\gamma^{\prime}(\tau)=0$. Then from $(6.72)$ we see that

$$
e(\tau, \tau-1)=\gamma(\tau) \theta(\tau-1) \varepsilon(\tau, \xi(\tau-1))=\varepsilon(\tau, \tau-1) .
$$

Similarly, letting $g=-e_{\tau}$, we have by (6.61), (6.53) and (6.72),

$$
\begin{aligned}
g(\tau, \tau-1) & =-\varepsilon_{\tau}(\tau, \tau-1)=f_{v}(1-\tau, \tau-1) \\
& =e^{1-\tau}+\left(1-e^{-(\tau-1)}\right) \exp \left[\alpha^{-1}(1-\tau+(\tau-1))\right]=\bar{p} .
\end{aligned}
$$

This means $S(\tau, \bar{p})=\tau-1$, so

$$
\begin{aligned}
e(\tau, \bar{p}) & =e(\tau, S(\tau, \bar{p}))=e(\tau, \tau-1) \\
& =\varepsilon(\tau, \tau-1)=f(1-\tau, \tau-1)=\alpha+(1-\alpha) e^{1-\tau} .
\end{aligned}
$$

Since (6.74) holds $\forall \tau>1-\delta / 2$,

$$
\partial e(\tau, p) /\left.\partial \tau\right|_{(\tau, \bar{p})}=(\alpha-1) e^{1-\tau} \quad(\tau>1-\delta / 2) ;
$$

in particular,

$$
\partial e(\tau, p) /\left.\partial \tau\right|_{(1, \bar{p})}=\alpha-1<-\bar{p} / 2,
$$

so WEAK fails at $(1, \bar{p})$.

6.3. Ideal gases. In this section we construct an example of an ideal gas $e$ for which no Hugoniot curve is monotone.

An ideal gas is a medium that satisfies (1.2)-(1.7) and

$$
p \tau=R T,
$$

where $R>0$ is a constant. In [1], (6.75), together with (1.2)-(1.7), is shown to be equivalent to

$$
e(\tau, S)=h(\tau H)
$$

where $H=\exp (-S / R)$. Here $h$ is a function defined on $(0,+\infty)$ satisfying:

$$
\begin{aligned}
& h \text { maps }(0,+\infty) \text { onto }(0,+\infty), \\
& h>0, h^{\prime}<0, h^{\prime \prime}>0, h^{\prime \prime \prime}<0, \\
& k \text { maps }(0,+\infty) \text { onto }(0,+\infty), \\
& k^{\prime}<0,
\end{aligned}
$$

where $k(x) \equiv-h^{\prime}(x) x$. In this situation we have

$$
p \equiv g(\tau, S)=-h^{\prime}(\tau H) H, \quad T=-R^{-1} h^{\prime}(\tau H) \tau H .
$$

By (6.81), $p \tau=-h^{\prime}(\tau H) \tau H=k(\tau H)$, so that

$$
e(\tau, p)=e(\tau, S)=\Psi(p \tau)
$$

where $\Psi=h \circ k^{-1}$. Thus $\Psi$ maps $(0,+\infty)$ onto $(0,+\infty)$ and $\Psi^{\prime}>0$. From 
this it is clear that $e$ for an ideal gas is an energy function that satisfies STRONG. Note that (6.82) can also be expressed,

$$
e(\tau, p)=h(x), \quad \text { where } p \tau=-h^{\prime}(x) x .
$$

We now give an example of an ideal gas for which every Hugoniot curve $H$ is not monotone, i.e., along $H, \tau$ is not a nonincreasing function of $p$. Let

$$
h(x)=e^{1 / x}-1
$$

Then

$$
h^{\prime}(x) x=-e^{1 / x} / x,
$$

and $h$ satisfies (6.77)-(6.80); therefore (6.83) defines an ideal gas. Fix $\tau_{0}$, $p_{0}>0$ and let $H$ be the Hugoniot curve with center $\left(\tau_{0}, p_{0}\right)$. Then $(\tau, p) \in H$ iff

$$
e(\tau, p)-e\left(\tau_{0}, p_{0}\right)=\frac{1}{2}\left(\tau_{0}-\tau\right)\left[p+p_{0}\right] .
$$

By (6.83), $(\tau, p) \in H$ iff

$$
h(x)-h\left(x_{0}\right)=\frac{1}{2}\left(\tau_{0}-\tau\right)\left[p+p_{0}\right]
$$

where

$$
p \tau=-h^{\prime}(x) x, \quad p_{0} \tau_{0}=-h^{\prime}\left(x_{0}\right) x_{0} .
$$

We show that along $H, \tau$ comes arbitrarily near $\tau_{0}$ as $p \rightarrow+\infty$. (See Lemma 4.1.) Suppose not. Then $\exists \delta, P>0$ such that along $H$,

$$
\tau_{0}-\tau>\delta \quad \text { whenever } p \geqslant P \text {. }
$$

Then we must have $x \rightarrow 0$ as $p \rightarrow+\infty$ along $H$. For otherwise, there would exist $\sigma>0$ and a sequence $\left\{p_{n}\right\}$ such that $p_{n} \rightarrow+\infty$ and, with $\left(\tau_{n}, p_{n}\right) \in H$ and $p_{n} \tau_{n}=-h^{\prime}\left(x_{n}\right) x_{n}(n=1,2, \ldots), x_{n} \geqslant \sigma \forall n$. Then by (6.77) and (6.78), for the values $x_{n}, \tau_{n}$ and $p_{n}$ the left member of (6.86) would be bounded as $n \rightarrow+\infty$ while the right member would approach $+\infty$ as $n \rightarrow+\infty$, a contradiction. Using (6.87), (6.86) becomes

$$
h(x)-h\left(x_{0}\right)=\frac{1}{2}\left(\tau_{0}-\tau\right)\left[-\frac{h^{\prime}(x) x}{\tau}-\frac{h^{\prime}\left(x_{0}\right) x_{0}}{\tau_{0}}\right] .
$$

Then by (6.84), (6.85) and the above, we get

$$
\begin{array}{r}
e^{1 / x} \geqslant e^{1 / x}-e^{1 / x_{0}}=\left(\tau_{0}-\tau\right)\left[\frac{e^{1 / x}}{2 x \tau}+\frac{e^{1 / x_{0}}}{2 x_{0} \tau_{0}}\right]>\left(\tau_{0}-\tau\right) \frac{e^{1 / x}}{2 x \tau} \\
\left(p>p_{0}\right) .
\end{array}
$$

Thus

$$
\tau>\tau_{0} /(2 x+1) \rightarrow \tau_{0} \quad \text { as } p \rightarrow+\infty
$$


which contradicts (6.88). Hence along $H, \tau$ comes arbitrarily near $\tau_{0}$ as $p \rightarrow+\infty$. In particular, $H$ is not monotone.

The author wishes to express his gratitude to Professor Joel A. Smoller for much encouragement, direction and advice that contributed to the completion of this work.

\section{REFERENCES}

1. R. Courant and K. O. Friedrichs, Supersonic flow and shock waves, Interscience, New York, 1948.

2. W. D. Hayes, Gasdynamic discontinuities, Princeton Univ. Press, Princeton, N. J., 1960.

3. P. D. Lax, Hyperbolic systems of conservation laws. II, Comm. Pure Appl. Math. 10 (1957), 537-566.

4. T. P. Liu, Shock waves in the nonisentropic gas flow, J. Differential Equations 22 (1976), 442-452.

5. J. A. Smoller, On the solution of the Riemann problem with general step data for an extended class of hyperbolic systems, Michigan Math. J. 16 (1969), 201-210.

Department of Mathematics, Iowa State University, Ames, Iowa 50011

Current address: 4447 Palo Verde, Lakewood, California 90713 